\title{
Simulación del tráfico en una vía expresa y análisis estadístico de los resultados
}

\author{
Pedro J. Torres Vega \\ Universidad de Lima. Lima, Perú \\ Correo electrónico: ptorres@ulima.edu.pe
}

Recibido: 24/4/2012 / Aprobado: 31/5/2012

\begin{abstract}
RESUMEN: El creciente aumento de vehículos produce serios problemas de congestión en los centros urbanos; esta situación genera interrogantes sobre los métodos de control de las variables de interés en esta problemática. El caso de estudio presentado es una aproximación al tema y se basa en un escenario hipotético (vía expresa) que se modela y simula con un enfoque microscópico, considerando el movimiento individual de los vehículos. Se presenta una metodología para el análisis estadístico de los resultados de la simulación.
\end{abstract}

Palabras clave: Simulación sistemas estocásticos / ingeniería de tráfico / análisis estadístico de resultados simulación / proyectos de simulación

\section{An expressway traffic simulation and statistical analysis of the results}

AвSTRACT: The increasing number of vehicles produces serious bottlenecks problems in urban areas; this situation generates questions about the variables control methods of interest in this problem. The study case presents an approach to the topic and is based on a hypothetical scenario (expressway), which it models and simulates with a microscopic approach, considering the individual movement of vehicles. It presents a methodology to perform the statistical analysis of the simulation results.

Keywords: Stochastic systems simulation / traffic engineering / statistics analysis of simulation output / simulation projects 


\section{INTRODUCCIÓN}

La variabilidad e incertidumbre son características inherentes en cualquier sistema que involucre personas y maquinarias. La simulación de procesos estocásticos en producción, logística y servicios se utiliza cada vez más en la investigación de sistemas de creciente complejidad y variabilidad, que no podrían ser estudiados y evaluados mediante técnicas analíticas convencionales.

El presente artículo muestra los principales aspectos en el modelado de un sistema básico de control del tráfico para simularlo y observar su comportamiento en el ritmo de arribos, tiempo de recorrido y velocidades, así como monitorear el tráfico, experimentar congestionamientos y evaluar medidas para controlar el exceso de velocidad. También se modela y evalúa el funcionamiento de semáforos en intersecciones.

Dado que las muestras aleatorias son realizaciones de variables aleatorias, entonces las estimaciones podrían diferir significativamente de las respuestas verdaderas para el modelo y haber una significativa probabilidad de obtener inferencias erróneas acerca del sistema bajo estudio. En este sentido, se ilustran los aspectos más relevantes que deben ser abordados en la configuración de un experimento de simulación, tales como la determinación del tiempo de simulación adecuado, el número de réplicas, el período de calentamiento, la evaluación de indicadores para someterlos a la prueba de normalidad y la aplicación de técnicas de reducción de varianza. Finalmente, se identifican y miden los principales indicadores de desempeño del sistema y se muestra una metodología para realizar el análisis estadístico de las respuestas del modelo respecto a las variables de interés y la comparación de escenarios. Estos aspectos aseguran la validez estadística de los indicadores para realizar la inferencia estadística con un determinado nivel de confianza.

\section{DESCRIPCIÓN DEL CASO HIPOTÉTICO}

Se desea simular el funcionamiento de una vía expresa en sus 2 direcciones y se han establecido algunos supuestos que describiremos a continuación. La entrada de los vehículos a la vía se produce solo en el inicio de cada dirección y no hay entradas laterales. La dirección uno tiene 2 salidas laterales y una tercera salida al final de la vía; la dirección 2 tiene una salida lateral y una salida al final de la vía, como se muestra en la figura 1. 
Figura 1

Esquema de vía expresa hipotética

Salida 1

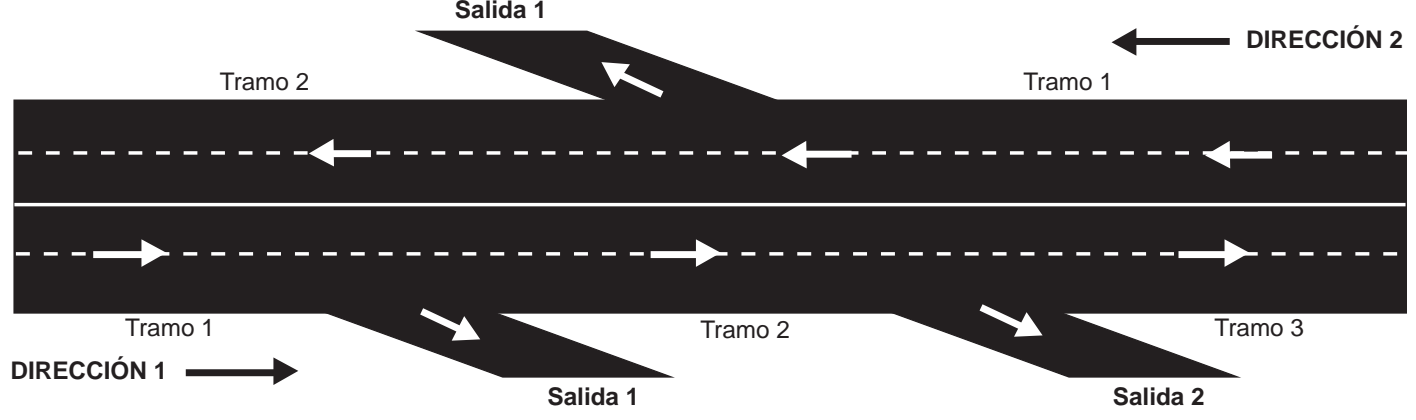

Elaboración propia.

Los vehículos llegan a la vía con un tiempo entre arribos que se ajusta a una distribución exponencial con una media de 40 segundos para la dirección uno y una media de 45 segundos para la dirección 2 . De los vehículos que viajan en la dirección uno, el 30\% se desvían por la salida 1 , de los que quedan y recorren el tramo 2 , el $60 \%$ toma la salida 2 , el resto continúa hasta el final de la vía. En la dirección 2 hay solo una salida lateral, el $30 \%$ toma la salida 1 y el resto de los vehículos llegan hasta el final. En el cuadro 1 se muestran los tiempos de recorrido y las probabilidades de arribo de los vehículos:

\section{Cuadro 1}

Tiempos de recorrido y probabilidades de arribo

\begin{tabular}{|c|c|c|c|c|}
\hline \multirow{2}{*}{$\begin{array}{c}\text { Rangos de } \\
\text { velocidades } \\
\text { (km/hora) }\end{array}$} & $\begin{array}{c}\text { Probabilidad } \\
\text { de arribo }\end{array}$ & $\begin{array}{c}\text { Tiempo de recorrido } \\
\text { (minutos) }\end{array}$ & $\begin{array}{c}\text { Probabilidad } \\
\text { de arribo }\end{array}$ & $\begin{array}{c}\text { Tiempo de recorrido } \\
\text { (minutos) }\end{array}$ \\
\hline hasta 60 & 0.30 & uniforme $(25,30)$ & 0.40 & uniforme $(22,26)$ \\
\hline $60-80$ & 0.50 & uniforme $(18,20)$ & 0.45 & uniforme $(16,19)$ \\
\hline más de 80 & 0.20 & uniforme $(14,18)$ & 0.15 & uniforme $(12,16)$ \\
\hline
\end{tabular}

Elaboración propia. 
En la dirección 1, los tiempos de recorrido para los tramos 2 y 3 corresponden al 80\% y 30\% del tiempo de recorrido del tramo 1, respectivamente. En la dirección 2, el tiempo de recorrido del segundo tramo es el $60 \%$ del tiempo de recorrido del tramo 1.

Al final de cada vía, existe un cruce o intersección en la que se ubica un semáforo, los tiempos de funcionamiento de los semáforos se muestran en el cuadro 2.

\section{Cuadro 2}

Tiempos de funcionamiento de los semáforos

\begin{tabular}{|l|c|c|c|c|}
\hline \multirow{2}{*}{ Tiempo } & \multicolumn{2}{|c|}{ Dirección 1 } & \multicolumn{2}{c|}{ Dirección 2 } \\
\cline { 2 - 5 } & Luz verde & Luz roja & Luz verde & Luz roja \\
\hline segundos & 300 & 100 & 240 & 120 \\
\hline
\end{tabular}

Elaboración propia.

Las condiciones iniciales para el experimento establecen que el semáforo de la dirección 1 iniciará con luz verde y el de la dirección 2 con luz roja, considerando despreciables los tiempos de arranque de los vehículos al inicio de la luz verde.

\section{MODELADO DEL SISTEMA, POR PROCESOS}

El modelo completo para el caso en estudio se muestra en las figuras 2 y 3 . 

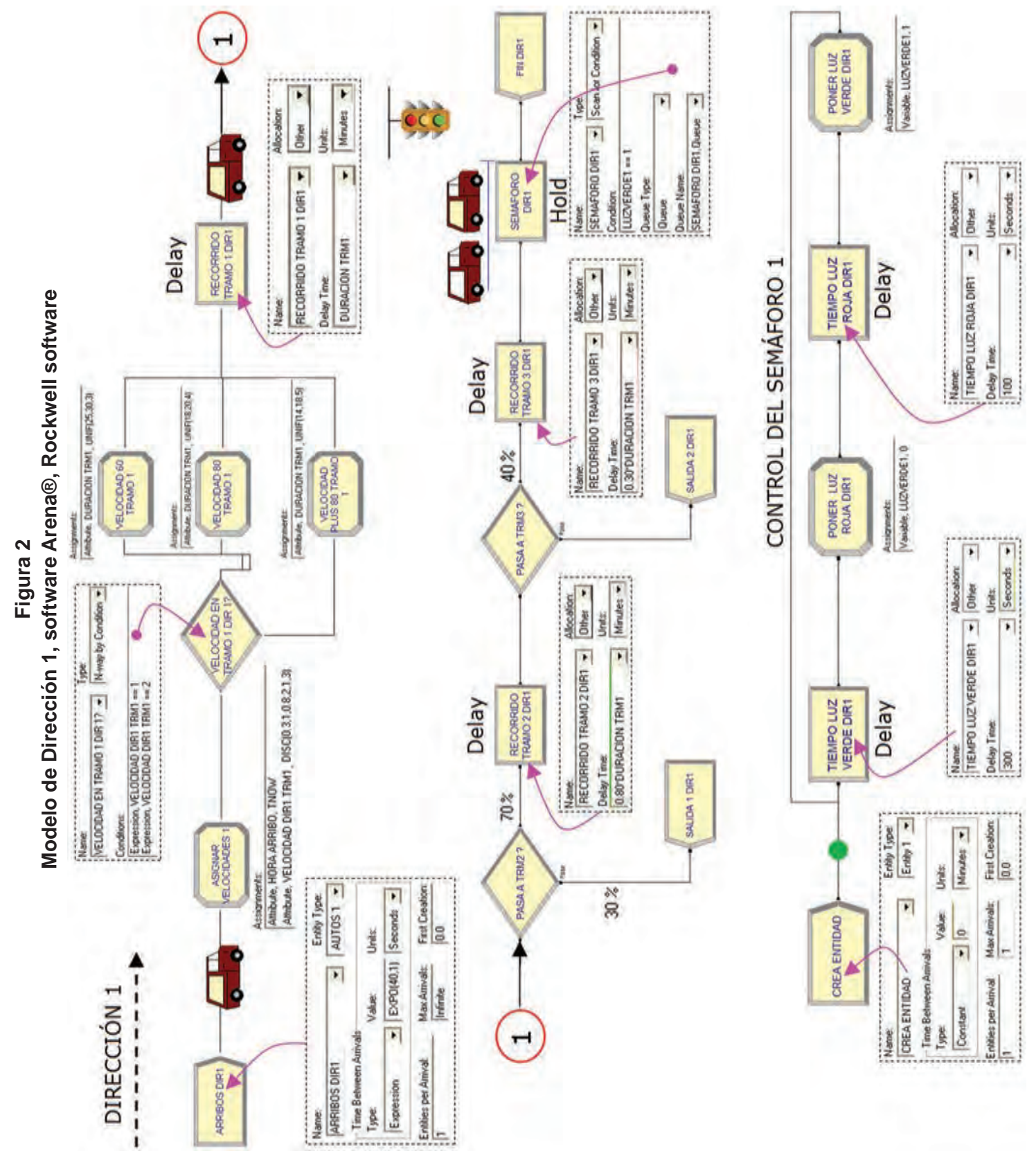

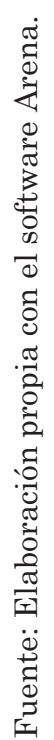




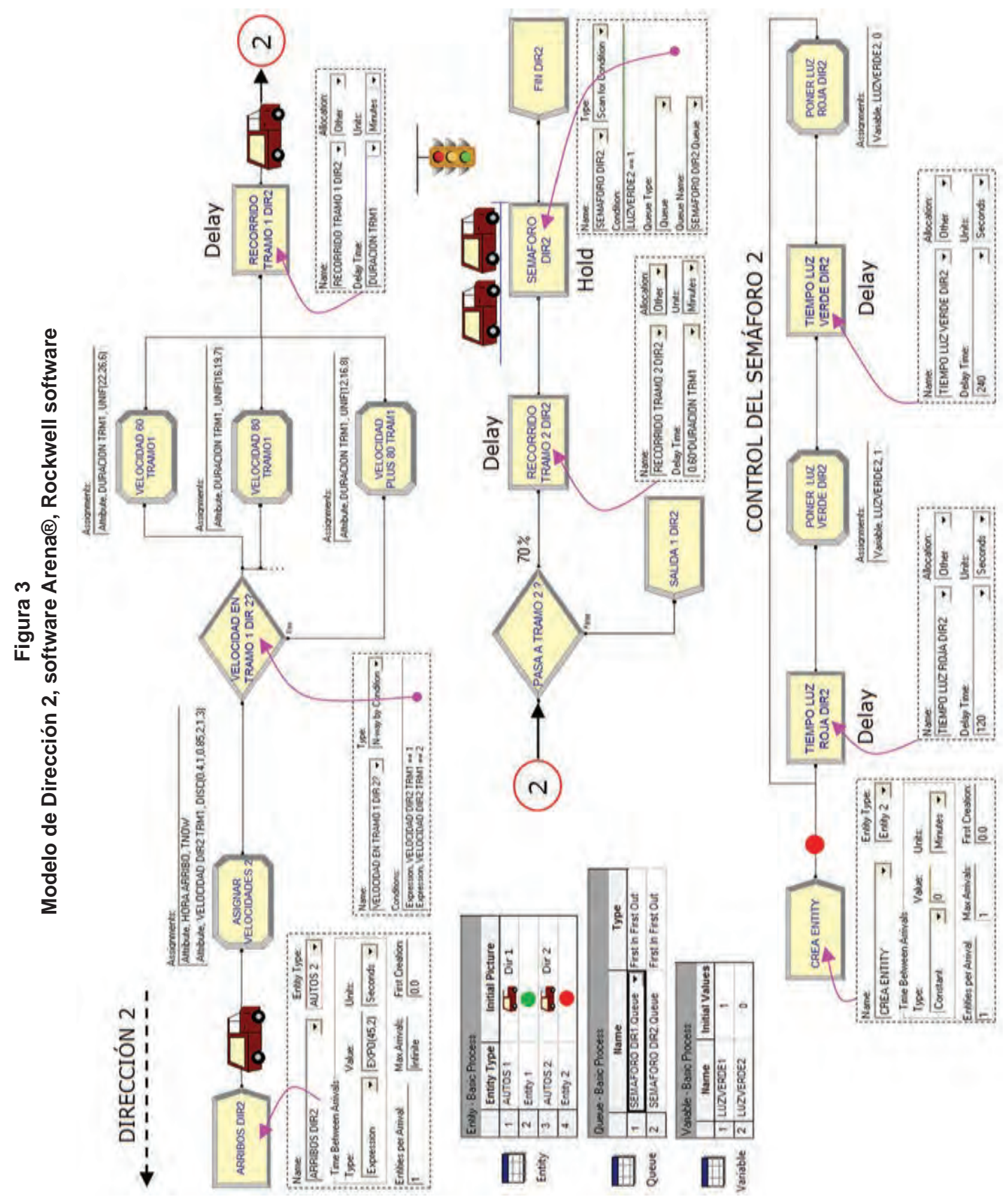

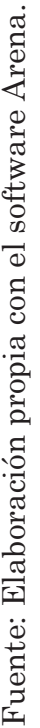




\section{CONFIGURACIÓN DEL EXPERIMENTO DE SIMULACIÓN}

El sistema en estudio corresponde a un sistema de estado estable, ya que el funcionamiento de una vía expresa se centra en el largo plazo, o no tiene término. En cuanto al tiempo de simulación, este debe ser el adecuado, si la simulación es muy corta los resultados pueden ser altamente variables; la simulación debe correr hasta que el intervalo de confianza del indicador medido se aproxime a la amplitud deseada. En nuestro experimento, consideraremos un período de ejecución igual a 60 horas de funcionamiento como punto de partida, el cual podría incrementarse para asegurarnos de que sea lo suficiente como para lograr los objetivos del estudio.

Como condiciones iniciales del experimento se plantea la suposición de que el sistema estará vacío al inicio de la simulación. Estas condiciones determinan un sesgo inicial que influye en el tiempo que lleva alcanzar la estabilidad en los resultados y en las estimaciones calculadas. El sistema alcanzará la estabilidad después de un cierto período de calentamiento o warm up (véase la figura 4) que es necesario determinar y eliminar de las estadísticas generales ya que distorsionan los resultados de los indicadores y los inclinan hacia valores inferiores en los momentos iniciales de la simulación, respecto de los que se obtendrían cuando se alcance la estabilidad.

Figura 4

Esquema período de calentamiento

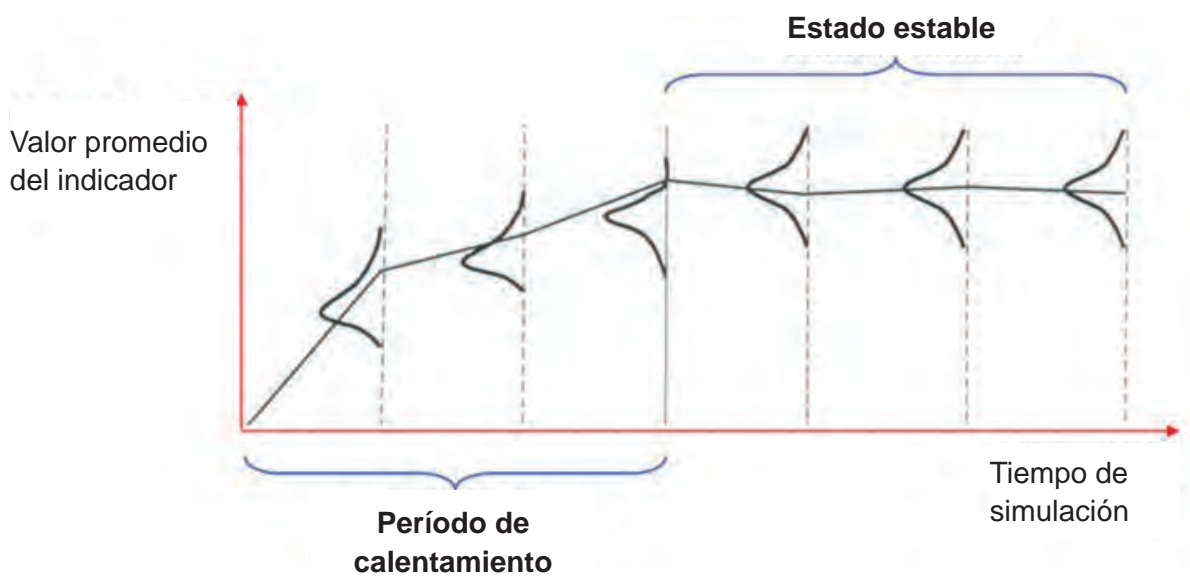

Fuente: Harrell (1995). 
Al eliminar de las estadísticas este período de calentamiento, los indicadores tienden a incrementar sus valores, ya que serán medidos a partir de haber alcanzado la estabilidad. Por lo tanto, se obtienen indicadores más precisos y cercanos con la realidad del sistema estable. Otra manera de anular este sesgo inicial es simular durante un período de tiempo muy largo.

En cuanto al número de réplicas, se determinará en función a una precisión de 0.1 minutos que se desea obtener en el tiempo de permanencia de los autos en la vía expresa (indicador de referencia). Dicha precisión es la mitad del ancho del intervalo o Half Width.

Los indicadores obtenidos de la simulación que son funciones lineales, bajo la suposición de que el muestreo (número de réplicas) es generada con probabilidades de selección iguales (equiprobable) de la muestra aleatoria, deberán estar normalmente distribuidos y se debe verificar que se cumpla la aplicación del teorema del límite central y así generalizar los resultados de la muestra hacia la población de estudio. Una referencia importante es la cantidad de observaciones por réplica, en todo caso, es necesario comprobar el ajuste de las muestras hacia la distribución normal mediante las pruebas Anderson-Darling, Kolmogorov-Smirnov y Chi-cuadrado.

\subsection{Detección visual aproximada del período de calentamiento}

Se ha considerado la evaluación de 3 indicadores: el número promedio de autos de inicio a fin de la vía en ambas direcciones, el tiempo de permanencia promedio para los 3 tramos de la dirección 1 y el tiempo entre salidas para la dirección 2 . El procedimiento empleado fue realizar una detección visual aproximada (véase la figura 5), revisando las gráficas en réplicas diferentes de los indicadores propuestos, observando y anotando los tiempos para alcanzar la estabilidad; finalmente, se consideró, de manera aproximada, el tiempo límite superior de las réplicas observadas. 
Figura 5

Gráficas representativas de los indicadores propuestos

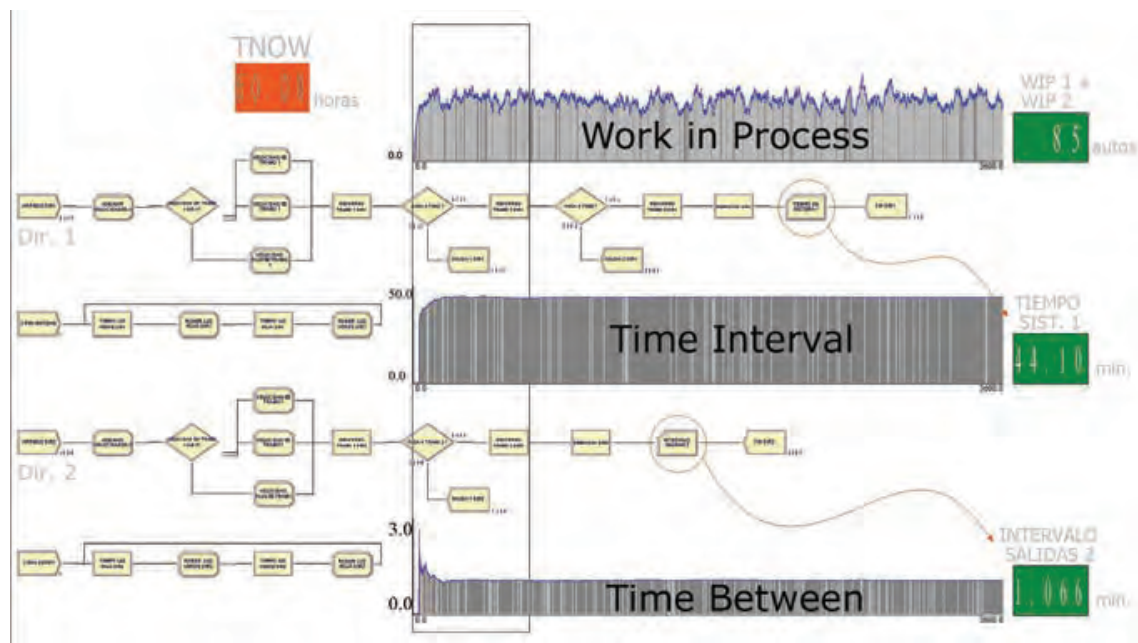

Elaboración propia con el software Arena.

\subsection{Determinación del número de réplicas de la simulación}

Si deseamos observar el comportamiento dinámico y general de los elementos del sistema en estudio es suficiente con realizar una sola corrida. Sin embargo, si deseamos obtener valores o mediciones estadísticamente válidas, debemos realizar varias réplicas independientes con distintos números aleatorios, que generarán distintos valores de las distribuciones muestreadas. Mediante la ejecución de múltiples replicaciones se obtienen respuestas independientes y valores medios de los resultados que se muestrean, así como la varianza y la desviación estándar que miden la dispersión de la variable aleatoria. El intervalo de confianza nos proporciona un grado de seguridad que podemos tener de la estimación, es decir, de la media del conjunto de respuestas. Para calcular el número de réplicas para nuestro experimento, tomaremos como referencia al tiempo promedio de permanencia en la dirección 1 , desde el inicio hasta el final de la vía y las consideraciones siguientes: 


\section{Figura 6}

Factor de precisión

Nivel de confianza propuesto: $1-\alpha=95 \%$

Probabilidad de error: $\alpha=5 \%$

Error deseado: e = máximo 0.1 minutos

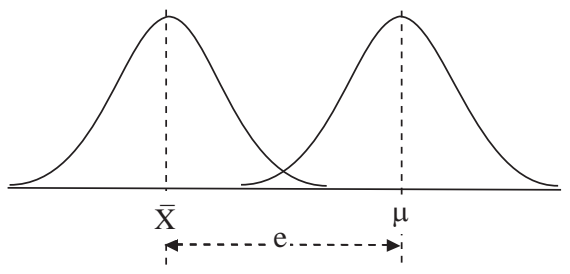

Elaboración propia.

El error e es un factor de precisión; es la mitad del ancho del intervalo o half-width. Es el error deseado entre la media estimada y la media teórica $\mu$ (figura 6). Existe una relación inversa entre error o mitad del ancho del intervalo $\mathbf{h}$ y el tamaño de $\mathbf{n}$ :

$$
h=t_{(n-1,1-\alpha / 2)} \sqrt{\frac{S_{n}{ }^{2}}{n}} h=Z_{(1-\alpha / 2)} \sqrt{\frac{S_{n}{ }^{2}}{n}}
$$

A mayor número de réplicas es menor el riesgo y el ancho del intervalo. En general, una manera de obtener intervalos más precisos, es aumentando el tamaño de la muestra $\mathbf{n}$. Consideremos arbitrariamente 20 muestras preliminares (figura 7): n': 20 réplicas.

\section{Figura 7}

Reporte de resultados

Replications: 20 Time Units: Minutes

\begin{tabular}{lrrrrrr|}
\hline User Specified & & & & & & \\
\hline Interval & Aversge & Half Wiath & $\begin{array}{r}\text { Minimum } \\
\text { Aversge }\end{array}$ & $\begin{array}{r}\text { Masimum } \\
\text { Aversge }\end{array}$ & $\begin{array}{r}\text { Minimum } \\
\text { Value }\end{array}$ & $\begin{array}{r}\text { Maximum } \\
\text { Value }\end{array}$ \\
\hline TIEMPO EN SISTEMA1 & 44.2788 & 0.14 & 43.6103 & 44.8102 & 29.4016 & 64.4948 \\
\hline
\end{tabular}

Fuente: Software Arena.

$\mathrm{Al}$ ejecutar las 20 réplicas al modelo, obtenemos un half-width preliminar: $h_{0}=0.14$ minutos. 
- Método 1: Cálculo del número de réplicas para el experimento.

$$
n=n^{\prime}\left(\frac{h_{o}}{e}\right)^{2} \quad n=20\left(\frac{0.14}{0.1}\right)^{2} \quad n=39 \quad \Rightarrow 40 \text { réplicas. }
$$

- Método 2

$$
n=\left[\frac{t_{\left(n^{\prime}-1,1-\alpha / 2\right)} * S_{\left(n^{\prime}\right)}}{e}\right]^{2}
$$

$\mathrm{t}_{\left(\mathrm{n}^{\prime}-1,1-\alpha / 2\right)}=2.093$ (valor de tabla: distribución t-Student)

$S_{n}=0.289$ (desviación estándar de la muestra preliminar n')

$\mathrm{Al}$ ejecutar 20 réplicas preliminares con un n.c. 95\% obtenemos el reporte de la figura 8.

Figura 8

Reporte de resultados

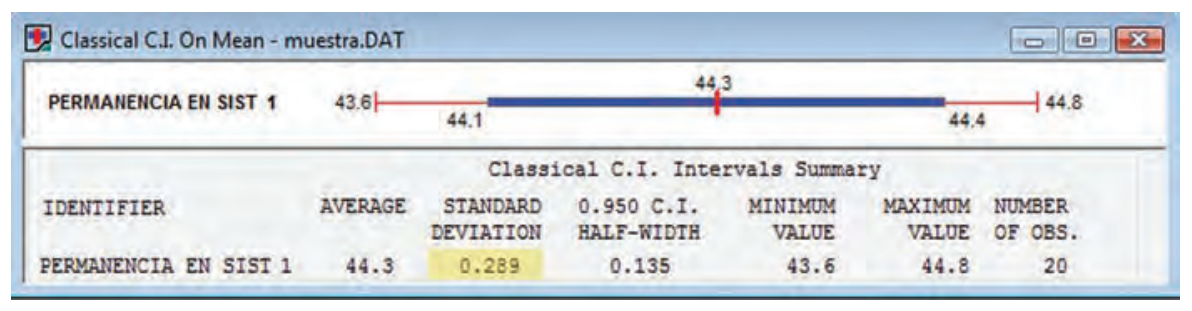

Fuente: Software Arena.

Reemplazando valores tenemos: $n=\left[\frac{2.093 * 0.289}{0.1}\right]^{2} \quad n=37$

La diferencia es mínima respecto al primer método. Para comprobar si se cumple con el factor de precisión propuesto, ejecutaremos el modelo por 40 réplicas y mediremos el indicador de referencia para un nivel de confianza de $95 \%$ (figura 9). 
Figura 9

Reporte de resultados

Replications: $40 \quad$ Time Units : Minutes

\section{User Specified}

\begin{tabular}{|c|c|c|c|c|c|c|}
\hline Interval & Average & Half Wiath & $\begin{array}{l}\text { Minimum } \\
\text { Average }\end{array}$ & $\begin{array}{l}\text { Maximum } \\
\text { Aversoge }\end{array}$ & $\begin{array}{c}\text { Minimum } \\
\text { Value }\end{array}$ & $\begin{array}{l}\text { Maximum } \\
\text { Value }\end{array}$ \\
\hline TIEMPO EN SISTEMA 1 & 44.2114 & 0.09 & 43.6103 & 44.8102 & 29.4016 & 64.5184 \\
\hline
\end{tabular}

Fuente: Software Arena.

Se aprecia un error de 0.09 minutos menor a la precisión requerida de 0.1 para el indicador.

De acuerdo con la figura 10, en lo sucesivo se considerará, como punto de partida, la configuración siguiente:

Figura 10

Configuración del experimento

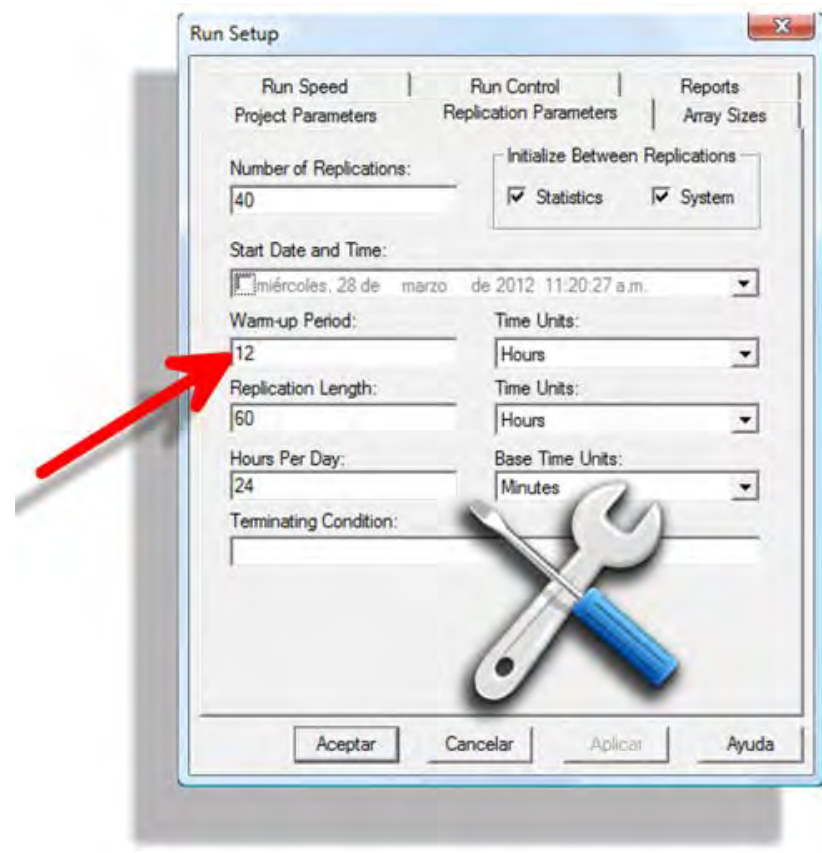

- El sistema inicialmente está vacío

- 40 réplicas para medir los indicadores de desempeño del sistema en estudio,

- 12 horas de período de calentamiento

- 60 horas de tiempo total de simulación

- 24 horas por día

- La base de tiempo está en minutos.

Fuente: Software Arena. 


\section{ANÁLISIS ESTADÍSTICO DE LOS INDICADORES DE DESEMPEÑO}

Durante la ejecución de la simulación se van registrando las mediciones individuales de la variable en estudio, el conjunto de observaciones de una réplica de simulación es una muestra perteneciente a alguna distribución de probabilidad. Cada muestra obtenida nos conduce a una "respuesta"; la estimación es el promedio muestral de un conjunto de repuestas. Una estimación también puede representar un valor total resultante de un conteo de observaciones, por ejemplo, el número de entidades en cola al final de la simulación.

$\mathrm{El}$ intervalo de confianza nos proporciona el grado de seguridad con que estamos estimando el valor medio de la distribución poblacional; el intervalo está asociado a un nivel de confianza que le podemos tener a esa estimación. Al ejecutar múltiples réplicas al modelo de simulación, el promedio de las respuestas está representado por $\overline{\mathrm{X}}$, que es el valor estimado de la media de la distribución. La dispersión de la variable aleatoria, respecto de su media, se mide mediante la desviación estándar s o la varianza $\mathbf{s}^{2}$.

$$
\overline{\mathrm{X}}=\frac{\sum_{i=1}^{n} x_{i}}{n} \quad s^{2}=\frac{\sum_{i=1}^{n}\left(x_{i}-\bar{X}\right)^{2}}{n-1}=\frac{\sum_{i=1}^{n} x_{i}^{2}-\frac{1}{n}\left(\sum_{i=1}^{n} x_{i}\right)^{2}}{n-1}
$$

$\mathrm{X}_{\mathrm{i}}$ : Es el valor o respuesta de la réplica i.

n: Es el número de réplicas del experimento de simulación.

El intervalo de confianza para la media poblacional, mediante las expresiones:

$$
\left[\bar{X} \pm t_{(n-1,1-\alpha / 2)} \frac{S_{n}}{\sqrt{n}}\right] \text { para } \mathrm{n}<30 \quad \mathrm{y} \quad\left[\bar{X}_{ \pm z_{(1-\alpha / 2)}} \frac{S_{n}}{\sqrt{n}}\right] \text { para } \mathrm{n}>30
$$

- Comparación de medias para muestras relacionadas: datos pareados Paired-t. 
Figura 11

Resultados posibles de la prueba Paired-t

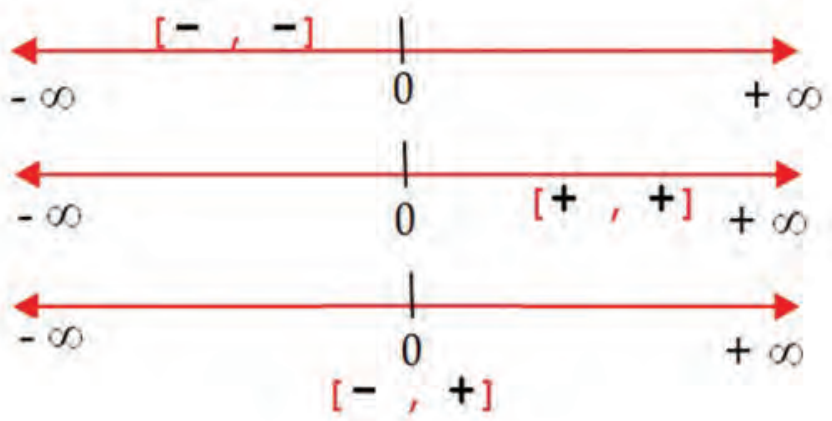

Elaboración propia.

Intervalo de confianza:

$$
\begin{aligned}
& {\left[\begin{array}{ll}
\bar{d} \pm t_{(n-1,1-\alpha / 2)} \frac{S_{n}}{\sqrt{n}}
\end{array}\right] \quad \begin{array}{l}
\bar{d}: \text { promedio de las diferencias } \\
S_{n}: \text { Desviación estándar de las }
\end{array}} \\
& \text { diferencias } \bar{d}=\bar{X}-\bar{Y} \quad \text { : }
\end{aligned}
$$

- Comparación de medias para muestras independientes: prueba Two-sample-t.

$$
\begin{aligned}
{\left[\bar{X}_{1}-\bar{X}_{2} \pm t_{\left(n_{1}+n_{1}-2,1-\alpha / 2\right)} S_{p} \sqrt{\frac{1}{n_{1}}+\frac{1}{n_{2}}}\right] } & S_{p}=\sqrt{\frac{\left(n_{1}-1\right) s_{1}^{2}+\left(n_{1}-1\right) s_{2}^{2}}{n_{1}+n_{2}-2}} \\
\mathrm{~S}_{\mathrm{p}}: & \text { Desviación estándar } \\
& \text { Ponderada (combinada). }
\end{aligned}
$$

\section{Indicador 1}

En este experimento se desea realizar con $95 \%$ de confianza, la estimación del indicador tiempo promedio de permanencia de los vehículos, que van desde el inicio hasta el final de cada vía. 
En la figura 12 se muestra el módulo, así como la configuración de la variable de interés:

Figura 12

Medición del indicador 1: Tiempo promedio de permanencia
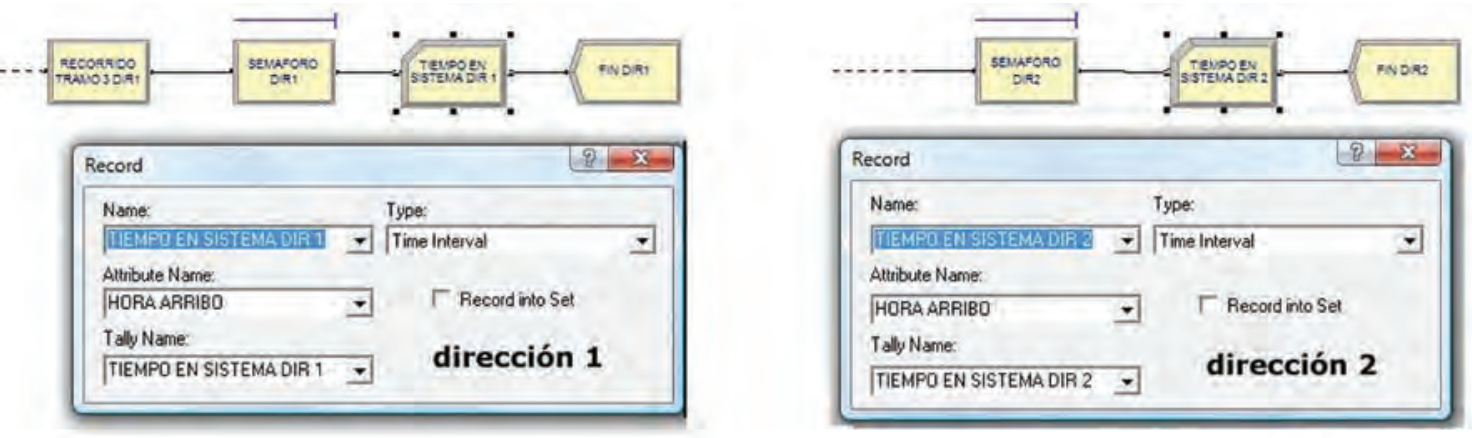

\begin{tabular}{|c|c|c|c|c|c|}
\hline \multicolumn{6}{|c|}{ Statistic-Advanced Process } \\
\hline & Name & Type & Expression & Report Label & Output File \\
\hline 1 & TEMPO PROM PERMANENCIA DR 1 & Output & TAVG (TEMPO EN SISTEMA DIR 1) & TEMPO PROII PERMANENCIA DR 1 & CITS DIR1,DAT \\
\hline 2 & TEMPO PROM PERIMANENCIA DR 2 & Output & TAVG(TEMPO EN SISTEMA DIR 2) & TEMPO PROM PERMANENCIA DR 2 & C:ITS DIR2.DAT \\
\hline
\end{tabular}

Elaboración propia con el software Arena.

Figura 13

Intervalos de confianza para el indicador 1

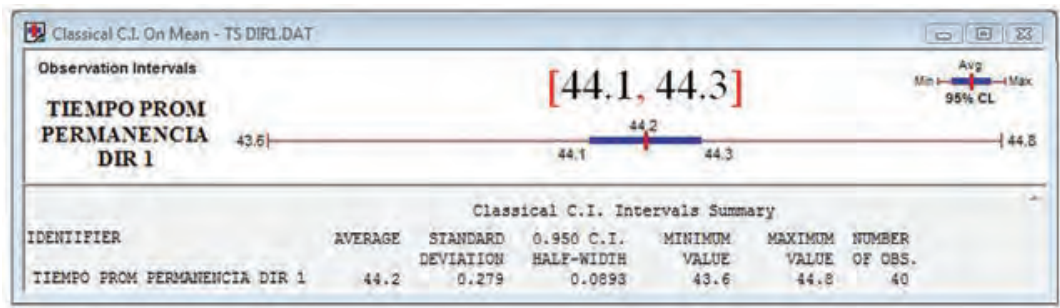

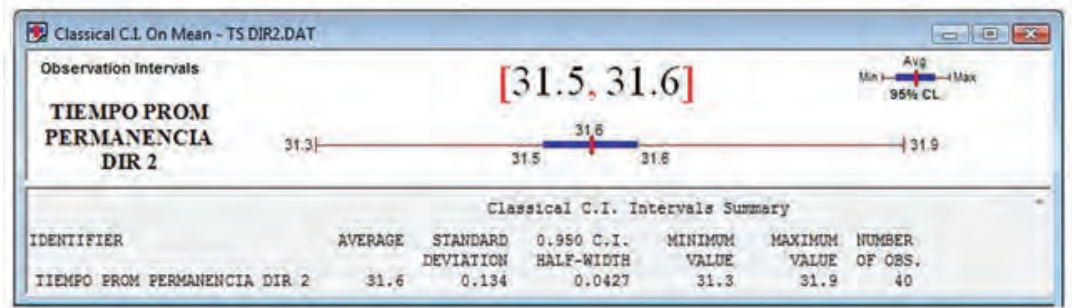

Fuente: Software Arena. 


\section{Conclusiones:}

Se ha estimado, con $95 \%$ de confianza, que el tiempo promedio de permanencia en la dirección 1 se encuentra en el intervalo: [44.1, 44.3] minutos. En la dirección 2 se está en el intervalo: [31.5, 31.6] minutos. El resultado es comprensible porque la dirección 1 está compuesta por 3 tramos, lo que implica un factor de 2.1 del tiempo promedio para cruzar la dirección 1 :

Tiempo total $=100 \%$ tramo $1+80 \%$ tramo $1+30 \%$ tramo 1 .

Para verificar la expresión anterior supongamos que el tiempo asignado a un vehículo en el tramo 1 es de 28 minutos, entonces, el tiempo que le demandará cruzar la vía completa será de $28+0.8 * 28+0.3 * 28=58.8$ minutos. Esta cifra también representa el producto de 28 por el factor 2.1. En cuanto a la dirección 2, al tener tiempos de recorrido cercanos a los de la dirección 1 y al tener solo 2 tramos, entonces posee un factor de 1.6 del tiempo promedio para cruzar la dirección 2 , menor al de la dirección 1. Si se aplicara la prueba de comparación de medias con la prueba two-sample-t para evaluar si existe significancia estadística, es evidente que se encontrarían diferencias significativas entre ambos promedios.

\section{Indicador 2}

Se desea realizar una comparación de medias con $90 \%$ de confianza, entre el número promedio de autos que transitaron por ambas vías $y$ analizar si existe diferencia significativa entre ambos indicadores.

Medición de la variable de interés: número promedio de autos o work in process (wip):

- Método 1: Medición del wip

Figura 14

Medición del indicador 2: Método 1

\begin{tabular}{|l|l|l|l|l|l|}
\hline \multicolumn{2}{|c|}{ Statistic-Advanced Process } & \multicolumn{1}{c|}{ Type } & \multicolumn{1}{c|}{ Expression } & Report Label & Output File \\
\hline & \multicolumn{1}{|c|}{ Name } & \multicolumn{1}{c|}{ Type } & \\
\hline 1 & NUM PROM1 & Time-Persistent & EntitiesWIP(AUTOS 1) & NUM PROM1 & \\
\hline 2 & WIP DIR 1 & Output & DAVG(NUM PROM1) & WIP DIR 1 & C:IWIP DIR1.DAT \\
\hline 3 & NUM PROM2 & Time-Persistent & EntitiesWIP(AUTOS 2) & NUM PROM2 & \\
\hline 4 & WIP DIR 2 & Output & DAVG(NUM PROM2) & WIP DIR 2 & C:IWIP DIR2.DAT \\
\hline
\end{tabular}

Elaboración propia con el software Arena. 
- Método 2: Medición del wip

En este caso utilizaremos una variable global "CUENTA" para contabilizar el número de autos en cualquier instante de la simulación (figura 15), así tenemos que:
cuenta $=$ cuenta +1
cuando ingresa un auto al sistema
cuenta $=$ cuenta -1
cuando egresa un auto del sistema

Figura 15

Modelo para indicador 2: Método 2
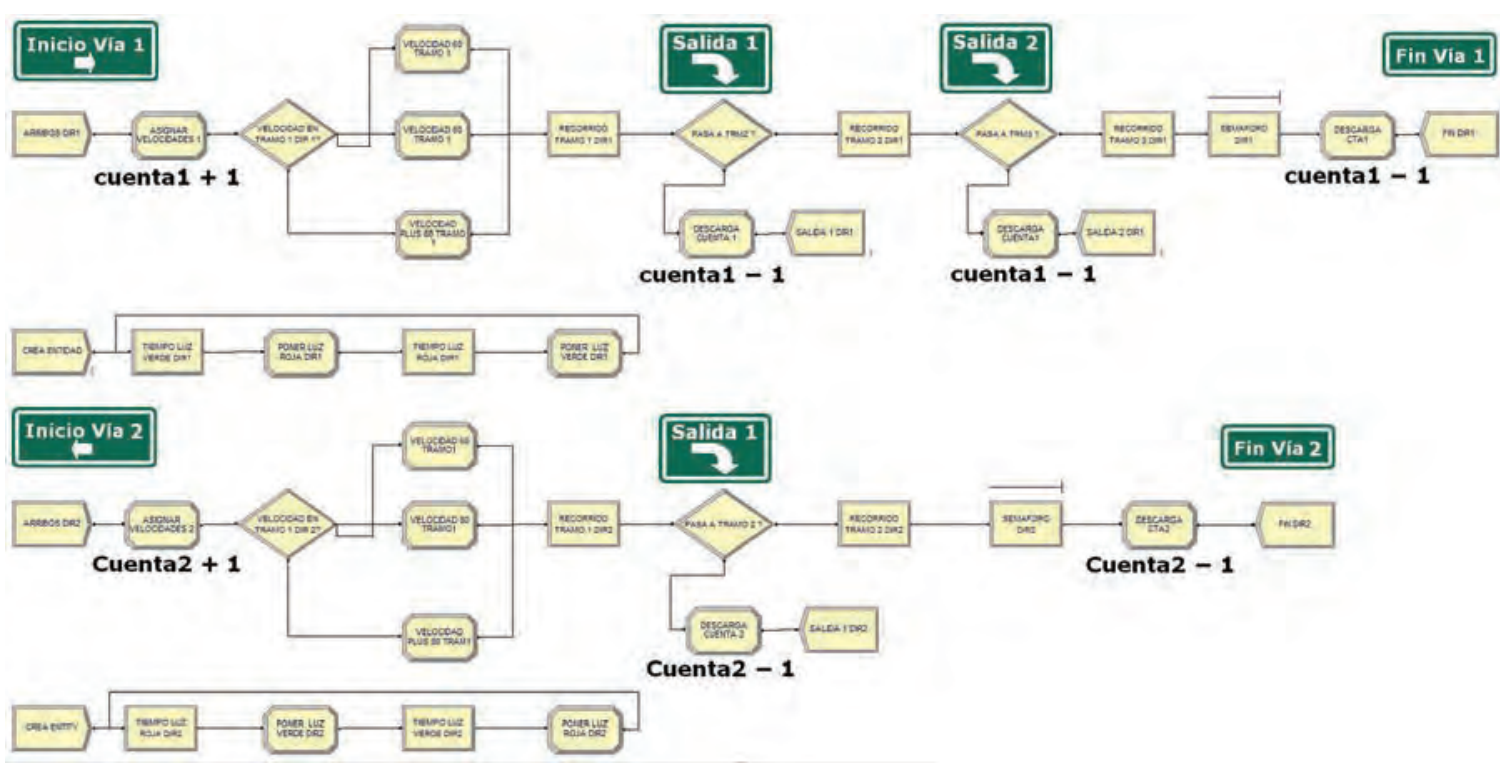

Elaboración propia con el software Arena.

Figura 16

Medición del indicador 2: Método 2

\begin{tabular}{|c|c|c|c|c|c|}
\hline \multicolumn{6}{|c|}{ Statistic - Advanced Process } \\
\hline & Name & Type & Expression & Report Label & Output File \\
\hline 1 & WIP DIR 1 & Output & DAVG(CUENTA1 Value) & WIP DIR 1 & C:IWIP DIR1.DAT \\
\hline 2 & WIP DIR 2 & Output & DAVG(CUENTA2 Value) & WIP DIR 2 & C:IWIP DIR2.DAT \\
\hline
\end{tabular}

Elaboración propia con el software Arena. 
Figura 17

Comparación de medias del indicador 2, wip: Two sample-t

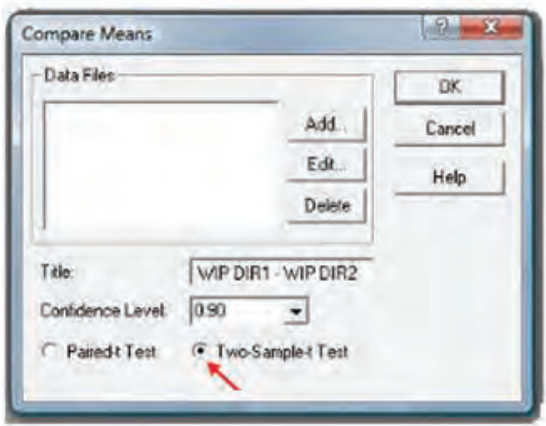

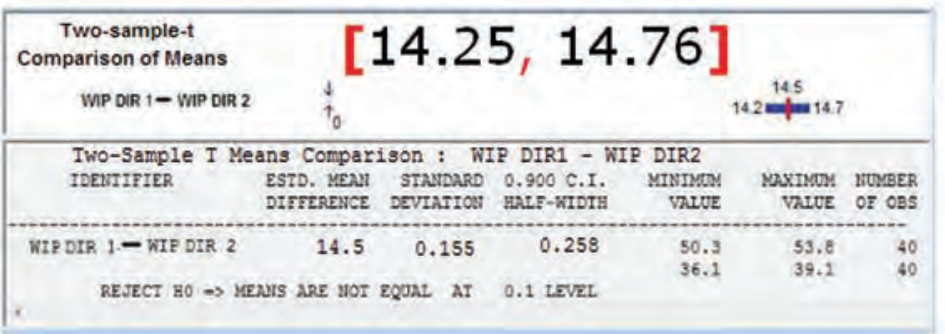

Elaboración propia con el software Arena.

\section{Conclusiones:}

Existe significancia estadística para el número promedio de vehículos que transitaron por ambas vías; la prueba arroja un intervalo: [14.25, 14.76] vehículos (figura 17). Existen diferencias estadísticamente significativas para aceptar la hipótesis alternativa $\mathrm{H}_{1}$, las medias son diferentes con $90 \%$ de confianza, la dirección 1 presenta un mayor promedio de vehículos. El resultado se explica por las diferencias en las tasas de llegada de los autos:

Tiempo entre arribos (segundos/autos)

Dirección 1: Exponencial (40)

Dirección 2: Exponencial (45)

\section{Tasa (autos/hora)}

$$
\begin{aligned}
& \frac{\text { autos }}{40 \mathrm{seg} .} * \frac{60 \mathrm{seg}}{\mathrm{min} .} * \frac{60 \mathrm{~min} .}{\text { hora }}=90 \frac{\text { autos }}{\text { hora }} \\
& \frac{\text { autos }}{45 \mathrm{seg} .} * \frac{60 \mathrm{seg}}{\mathrm{min} .} * \frac{60 \mathrm{~min} .}{\text { hora }}=80 \frac{\text { autos }}{\text { hora }}
\end{aligned}
$$

El resultado también se explica por la diferencia entre el número de tramos que poseen ambas vías. La dirección 1 tiene un factor de 2.1 mientras que la dirección 2 posee un factor de 1.6.

\section{Indicador 3}

Se desea experimentar la adición de tres rangos de velocidades en ambas direcciones, las cuales se ajustan a distribuciones de probabilidad uniforme $(40,60)$, uniforme $(60,80)$ y uniforme $(80,100)$ en kilómetros 
por hora, cada una se asocia a la duración ya existente en el tramo uno. Se desea evaluar con un nivel de confianza de $90 \%$ si existe diferencia estadisticamente significativa entre las velocidades promedio empleadas en las dos direcciones.

En la figura 18 se muestran los cambios en el modelo, de acuerdo a la situación planteada.

Figura 18

Cambios en el modelo y medición del indicador 3: Velocidad promedio

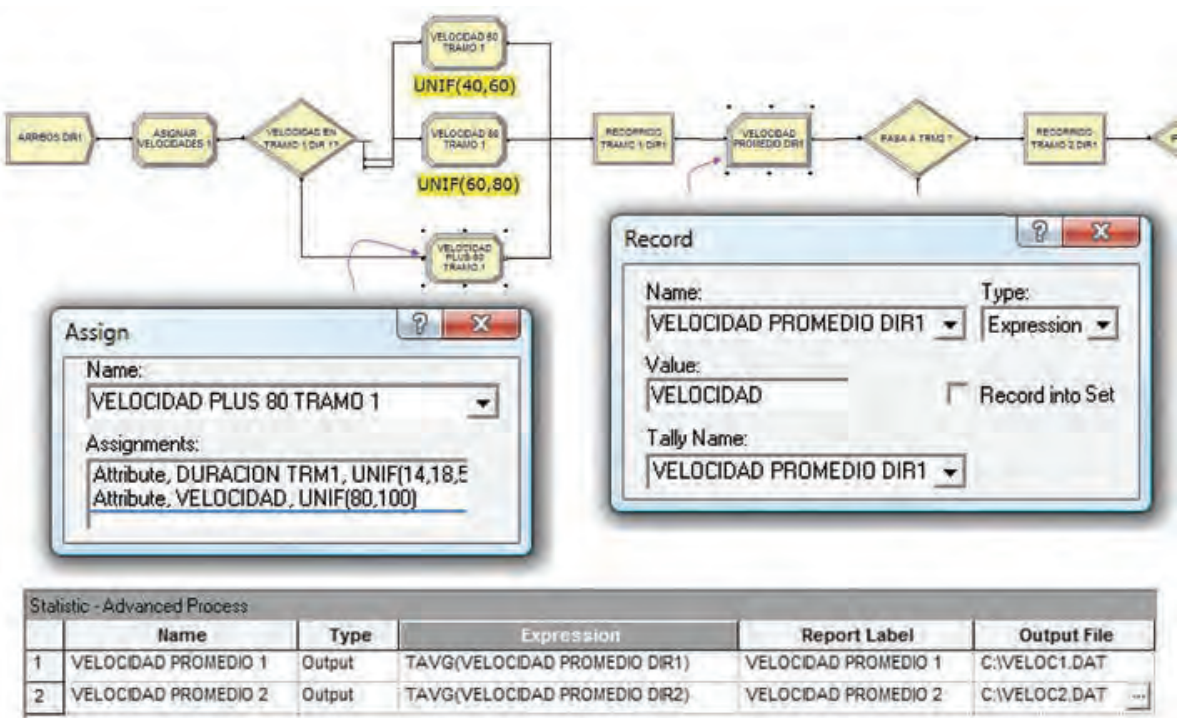

Elaboración propia con el software Arena.

Figura 19

Comparación de medias del indicador 3: Two sample-t

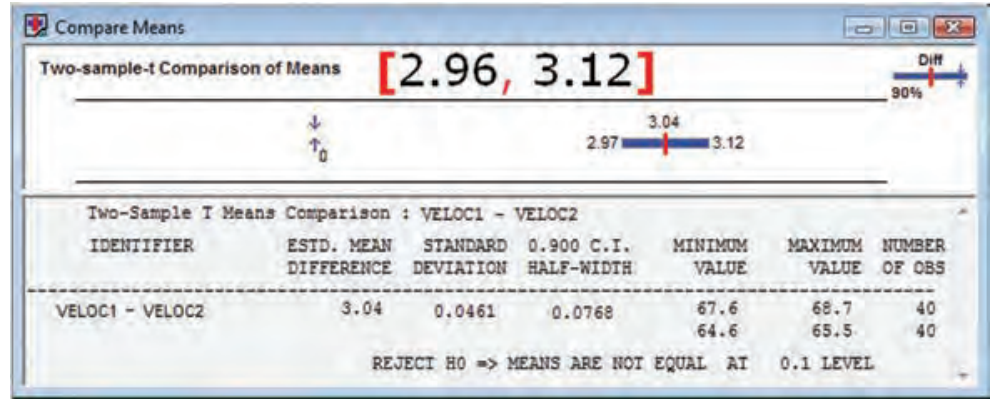

Fuente: Software Arena. 


\section{Conclusiones:}

Existe significancia estadística entre las velocidades promedio que los vehículos emplean en ambas direcciones; la prueba arroja un intervalo: [2.96, 3.12] kph (figura 19). Con 90\% de confianza las medias no son iguales, se rechaza la hipótesis nula $\mathrm{H}_{0}$, la dirección 1 presenta una velocidad promedio mayor. Si bien las vías son totalmente independientes, la diferencia se explica principalmente por las distintas proporciones de las probabilidades vinculadas a las velocidades en la dirección $1(30 \%, 50 \%$ y $20 \%)$ y en la dirección $2(40 \%, 45 \%, 15 \%)$. En general, existe una relación inversa entre velocidad promedio y tiempo de viaje, pero el factor determinante para esta relación son las proporciones de las probabilidades mencionadas. Para comprobar esta afirmación se colocó la misma proporción de las probabilidades para ambas direcciones: $30 \%, 50 \%$ y $20 \%$. Planteado de esta manera, no existen diferencias significativas entre las velocidades promedio: $[-0.1290,+0.0803] \mathrm{km} / \mathrm{h}$. También se concluye que el tiempo entre arribos de los autos no es un factor influyente.

\section{Indicador 4}

Se desea investigar la posibilidad de mejorar el tiempo promedio de espera de los autos, en el semáforo de la dirección 1, reduciendo el tiempo de luz verde de 300 a 240 segundos.

Figura 20

Cambio en el modelo y medición del indicador 4, tiempo promedio de espera

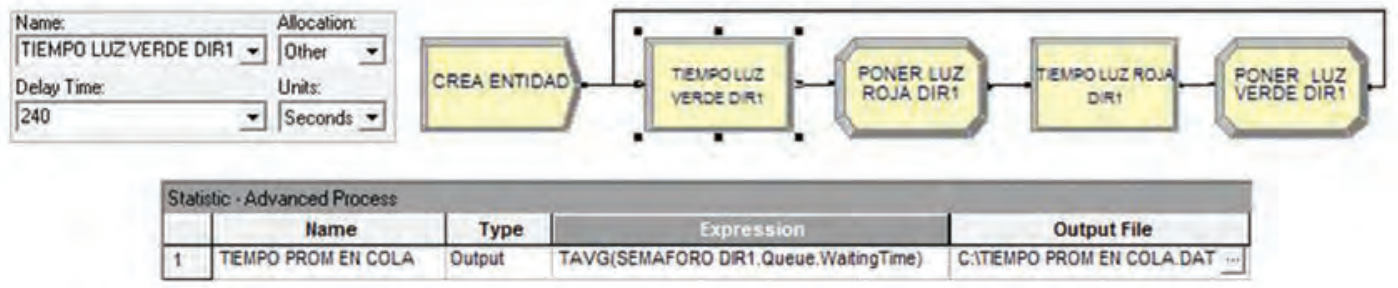

Elaboración propia con el software Arena. 


\section{Técnicas de reducción de varianza}

En cada réplica del modelo se utilizan diferentes secuencias de números e independientes entre sí. Las técnicas de reducción de varianza explotan la capacidad de control de los generadores de números aleatorios que conducen la simulación. Normalmente reutilizamos dichos números aleatorios para inducir correlaciones favorables que reduzcan el ruido en el output.

- Técnica de secuencias comunes

Esta técnica utiliza las mismas series de números aleatorios en experimentos sucesivos de comparación de escenarios. Se trata de obtener una reducción de varianza de la diferencia a partir de la sincronización de las mismas series de números para distintas configuraciones. A partir de una semilla original los sistemas serán comparados en las mismas circunstancias.

$$
\text { Si Z }=\mathrm{X}-\mathrm{Y}==>\operatorname{Var}(\mathrm{Z})=\operatorname{Var}(\mathrm{X})+\operatorname{Var}(\mathrm{Y})-2 \operatorname{Cov}(\mathrm{X}, \mathrm{Y})
$$

Si la $\operatorname{Cov}(\mathrm{X}, \mathrm{Y})$ es grande, por haber introducido correlación positiva entre ambas series, entonces la varianza de la diferencia: $\operatorname{Var}(\mathrm{Z})$, será mucho menor. En la formulación del modelo original se utilizaron secuencias comunes, tal como se indica en el cuadro 3.

\section{Cuadro 3}

Secuencias de números aleatorios utilizadas

\begin{tabular}{|c|c|c|}
\hline & \multicolumn{2}{|c|}{ SECUENCIAS DE NÚMEROS ALEATORIOS } \\
\hline & Dirección 1 & Dirección 2 \\
\hline Arribos & secuencia 1 & secuencia 2 \\
\hline
\end{tabular}

\begin{tabular}{|c|c|c|}
\hline Velocidades & $\begin{array}{c}\text { Tiempo de recorrido } \\
\text { tramo 1 }\end{array}$ & $\begin{array}{c}\text { Tiempo de recorrido } \\
\text { tramo 1 }\end{array}$ \\
\hline Hasta 60 & secuencia 3 & secuencia 6 \\
\hline $\mathbf{6 0}-\mathbf{8 0}$ & secuencia 4 & secuencia 7 \\
\hline más de 80 & secuencia 5 & secuencia 8 \\
\hline
\end{tabular}

Elaboración propia. 
Figura 21

Resultados del indicador 4: Secuencias comunes

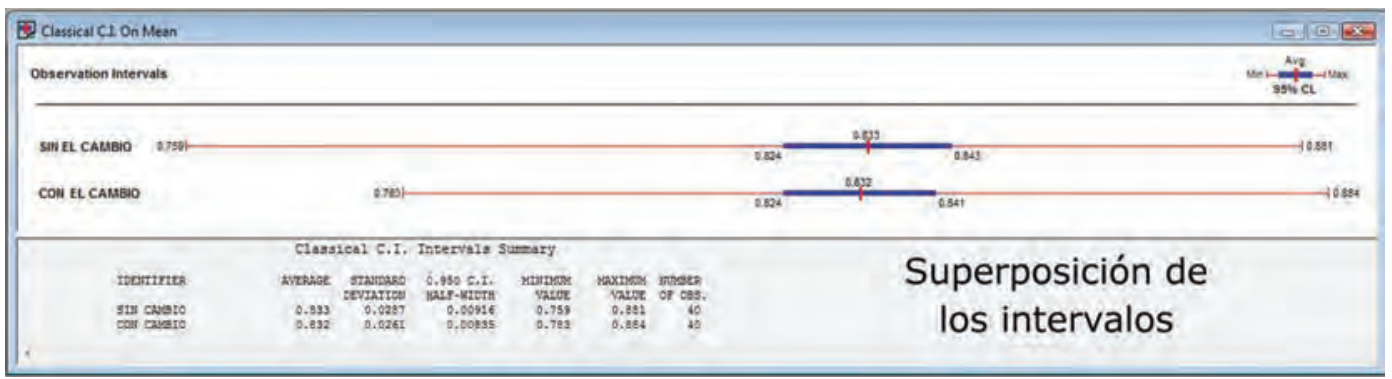

Fuente: Software Arena.

Figura 22

Comparación de medias con secuencias comunes: Prueba Paired-t.
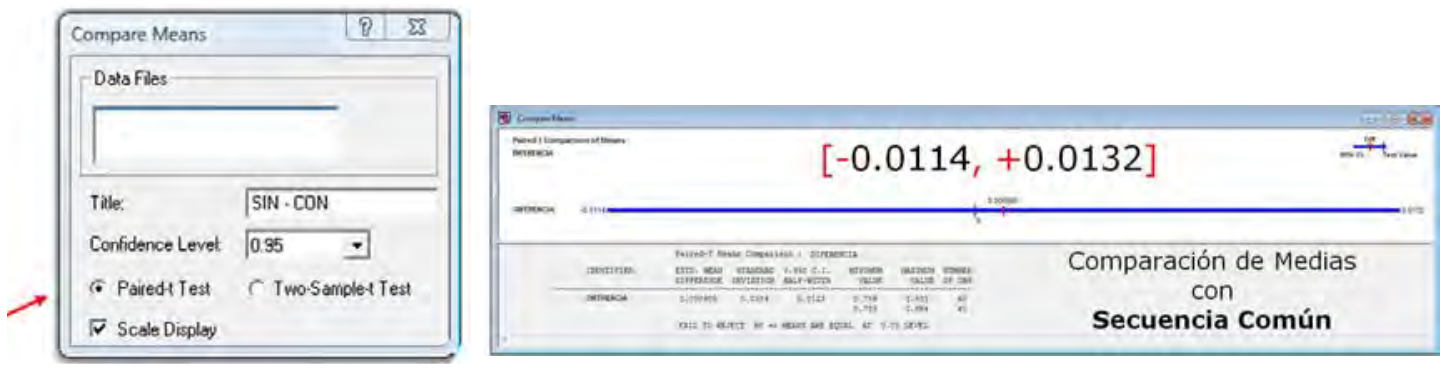

Fuente: Software Arena.

- Técnica de secuencias antitéticas

El método consiste en ejecutar una primera réplica con la serie de números aleatorios $u_{i}(i=1,2, \ldots n)$. Luego, se ejecuta una segunda réplica con los valores antitéticos $\left(1-u_{i}\right)(i=1,2, \ldots n)$. Este muestreo complementario induce una correlación negativa entre las respuestas de ambas simulaciones. En principio, ambas "se compensan" y se conjetura que las respuestas $\mathrm{y}(1)$ e $\mathrm{y}(2)$ estarán correlacionadas negativamente, por lo que la varianza de sus promedios decrece. Tenemos $\mathrm{n} / 2$ promedios de parejas (y(i), $\mathrm{y}(\mathrm{i}+1))$, lo que nos conduce a la expresión de la varianza de $\mathrm{Y}=\mathrm{Y}(\mathrm{i})+\mathrm{Y}(\mathrm{i}+1)$ :

$$
\operatorname{Var}(\mathrm{Y})=1 / 4[\operatorname{var}(\mathrm{Y}(\mathrm{i}))+\operatorname{var}(\mathrm{Y}(\mathrm{i}+1))+2 \operatorname{Cov}(\mathrm{Y}(\mathrm{i}), \mathrm{Y}(\mathrm{i}+1)]
$$

Si las réplicas son independientes desaparecen los términos de covarianza. Sin embargo, si hacemos negativa la suma de covarianzas, 
podemos producir una varianza menor de la que ofrecen réplicas independientes. En la figura 23 se realiza la aplicación del método antitético.

Figura 23

Inclusión del módulo Seeds: Secuencias antitéticas

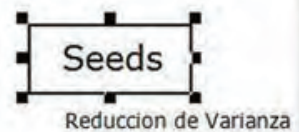

\begin{tabular}{|c|c|c|}
\hline Seeds & 8 & $x$ \\
\hline Identifier: & REDUCCION DE VARIANZA & - \\
\hline Seed Value: & 14561 & \\
\hline Initialize Option: & Antithetic & - \\
\hline
\end{tabular}

Fuente: software Arena.

Figura 24

Intervalos individuales del indicador 4: Secuencias antitéticas

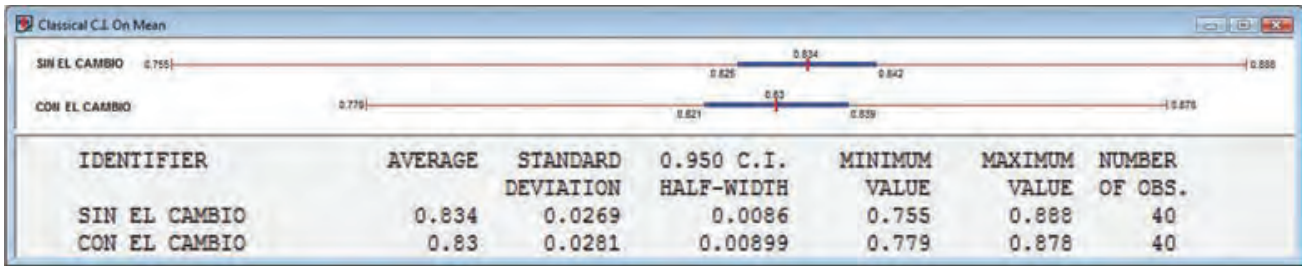

Fuente: software Arena.

Figura 25

Comparación de medias con secuencias antitéticas: Prueba Paired-t
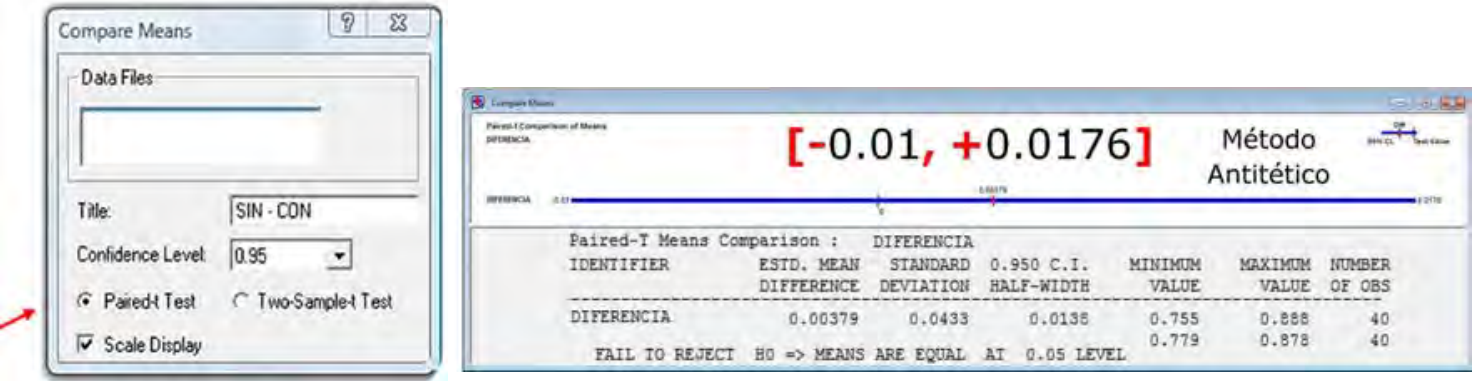

Fuente: software Arena. 


\section{Conclusiones:}

$\mathrm{Al}$ aplicar la comparación de medias utilizando la técnica de secuencias comunes se obtuvo el intervalo: [-0.0114, +0.0132] minutos (figura 22) y con secuencias antitéticas el intervalo: $[-0.0100,+0.0176]$ minutos (figura 25). En ambos casos, no existe evidencia estadística suficiente para aceptar la hipótesis alternativa $\mathrm{H}_{1}$ (es decir, para rechazar la hipótesis nula $\mathrm{H}_{0}$ ), las medias del indicador son iguales, con un $95 \%$ de confianza. La reducción en el tiempo de luz verde en el semáforo no genera ninguna mejora en cuanto a disminuir el tiempo promedio de espera de los vehículos. Este resultado es explicable, ya que la variable que tiene relación directa con el indicador medido es el tiempo de luz roja.

Se aplicaron técnicas de control de secuencias de números aleatorios para introducir correlación entre las diferentes réplicas y así reducir la varianza de los resultados y lograr mejores estimaciones e intervalos de confianza más precisos, sin tener que incrementar el tamaño de la muestra.

\section{EVALUACIÓN DE ESCENARIOS}

\section{Escenario 1}

Se desea investigar la posibilidad de implementar un servicio de asistencia vehicular, que inicialmente funcionará solo en la dirección 2. Contará con una camioneta de auxilio mecánico, cuya base será ubicada al inicio de la vía. Así, al ser requerida, esta se desplazará hasta el vehículo averiado, en un tiempo que se ajusta a una distribución uniforme (4,6) minutos. Asistir al auto averiado varía de acuerdo a una distribución uniforme $(10,30)$ minutos; finalmente, la camioneta regresa a su base. El mayor número de incidencias se produce después de los primeros $2 / 3$ de recorrido del tramo 2; cualquier vehículo tiene una probabilidad de 3\% de quedar averiado, en ese caso, el vehículo queda a un costado, a la espera del auxilio mecánico; una vez reparado el auto, este continúa con el último tercio que le resta de su recorrido en el tramo 2. Se desea estimar con $95 \%$ de confianza el número promedio de autos atendidos por el servicio de auxilio mecánico.

La figura 26 presenta la sección del modelo en la que se implementa el servicio de asistencia vehicular, véase además la configuración del indicador número de autos atendidos. 


\section{Figura 26}

Cambios en el modelo para incluir servicio de asistencia vehicular: Escenario 1

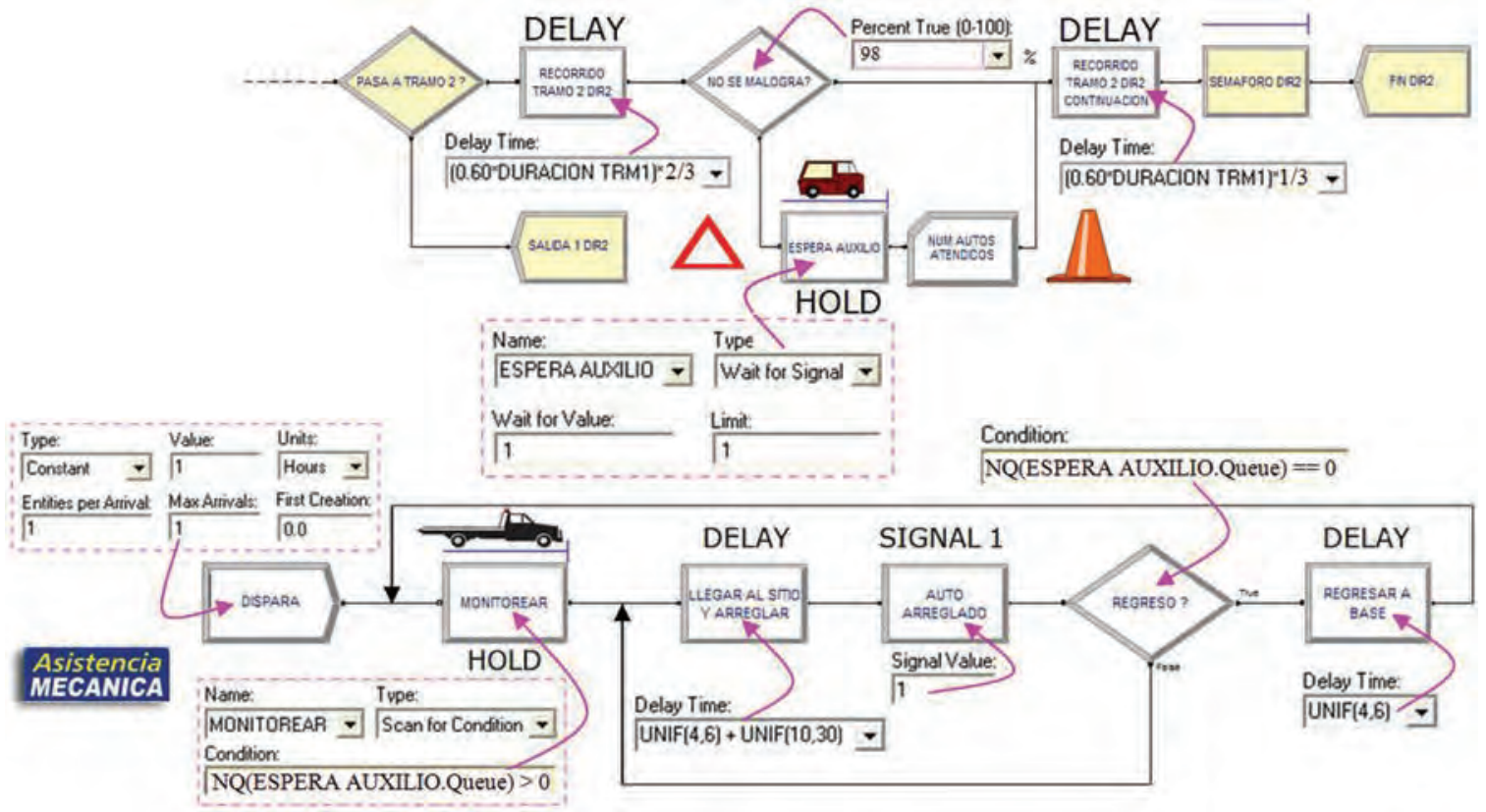

Elaboración propia con el software Arena.

Figura 27

Medición del indicador: Número de autos atendidos

\begin{tabular}{|c|c|c|c|c|}
\hline \multicolumn{5}{|c|}{ Statistic-Advanced Process } \\
\hline & Name & Type & Expression & Output File \\
\hline 1 & AUTOS ATENDIDOS & Output & NC(NUM AUTOS ATENDIDOS) & C:LAUTOS REPARADOS.DAT \\
\hline
\end{tabular}

Elaboración propia con el software Arena. 
Figura 28

Intervalo de confianza para el número de autos atendidos

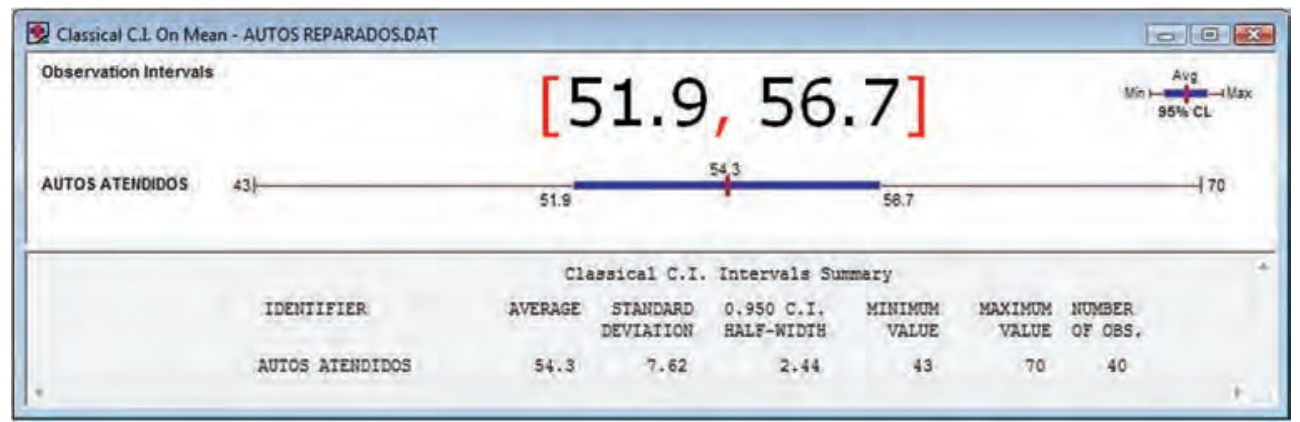

Fuente: Software Arena.

El intervalo de confianza para el indicador número de vehículos atendidos por el servicio de auxilio mecánico es: [51.86, 56.74] autos. Existe el indicio de que el indicador no pasaría el test de normalidad, por insuficiencia en el número de observaciones por muestra o réplica. Lo cual hay que confirmarlo. Los valores de las observaciones del indicador medido deben ajustarse a una distribución de probabilidad normal para poder realizar inferencia estadística a dicho indicador, de lo contrario lo deshabilitaría para tal propósito, pero no al modelo ni a otros indicadores. El teorema del límite central nos asegura que es posible realizar inferencia estadística al indicador si n es suficientemente "grande", es decir, si el tamaño de la muestra tiene un valor significativo (en nuestro caso, mayor que 70 observaciones por réplica).

\section{Prueba de normalidad}

El test consiste en someter las muestras obtenidas a pruebas estadísticas de bondad de ajuste a la distribución normal. Se aplicarán las pruebas de Chi-cuadrado y de Kolmogorov-Smirnov. 
Figura 29

Prueba de normalidad para el número de autos atendidos

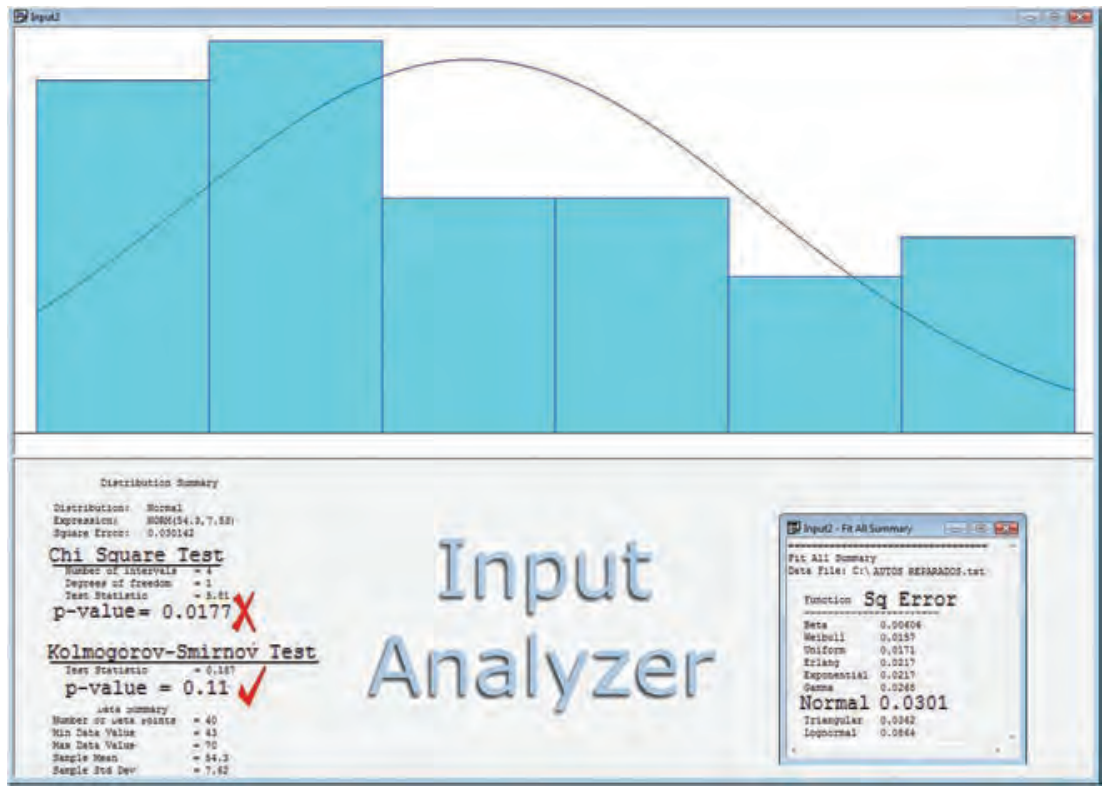

Fuente: Software Arena.

Nuestro indicador no pasa la prueba de normalidad para el test de Chi-cuadrado, arroja un valor $\mathrm{p}=0.0177$, menor que el valor del riesgo 0.05 (5\%); sin embargo, sí pasa el test de K-S. En todo caso, el ajuste es muy pobre y se puede apreciar la asimetría respecto a la media. En estas condiciones no es posible establecer un intervalo de confianza para $\mu$ (media teórica) y no será posible realizar inferencia estadística al indicador en estudio. Lo ideal es que el indicador pase ambas pruebas: Chi-cuadrado y K-S.

$\mathrm{Al}$ simular durante 60 horas el número de observaciones por réplica es insuficiente; aumentaremos progresivamente el tiempo de simulación de 10 en 10 horas hasta 100 y para cada caso repetiremos la prueba hasta obtener un tiempo adecuado con el que se logre pasar el test de normalidad para ambas pruebas. 
Figura 30

Prueba de normalidad: Aumento progresivo del tiempo de simulación

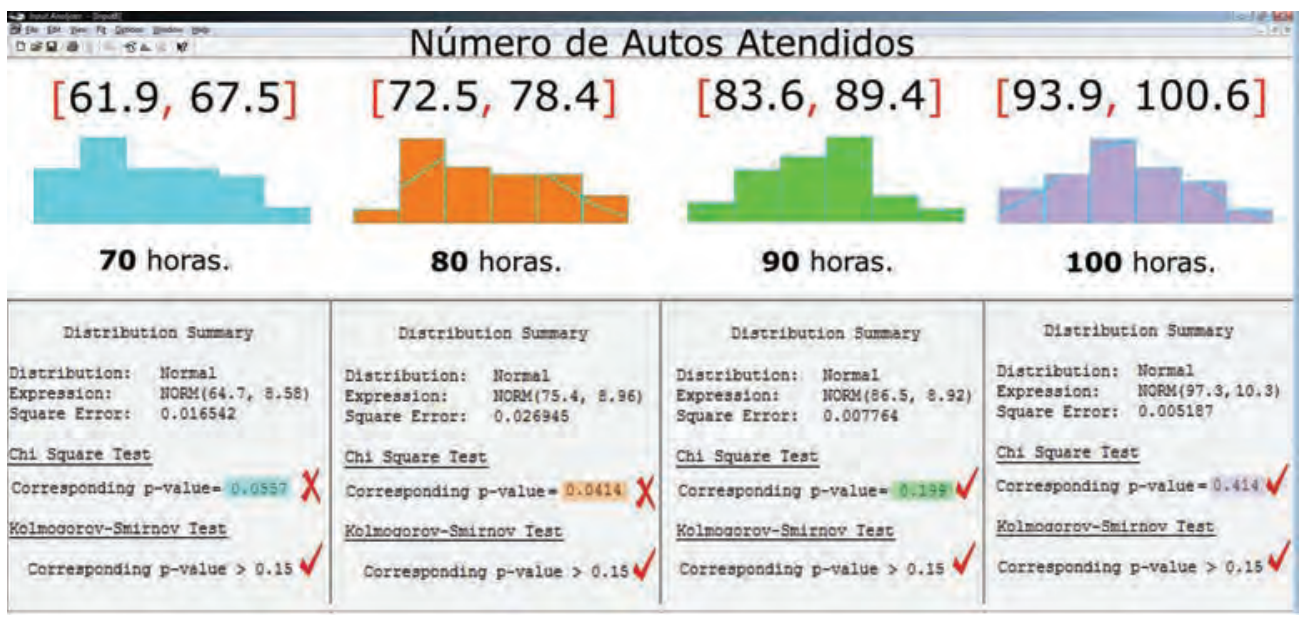

Elaboración propia con el software Arena.

\section{Conclusiones:}

Al repetir el experimento y aumentar progresivamente el tiempo de simulación (figura 30), aumentó el número de autos atendidos por réplica. En el cuadro 4 se muestran los resultados que arroja la prueba Chi-cuadrado.

Cuadro 4

Valores del indicador p. prueba Chi-cuadrado

\begin{tabular}{|c|c|c|c|c|c|}
\hline Horas & $\mathbf{6 0}$ & $\mathbf{7 0}$ & $\mathbf{8 0}$ & $\mathbf{9 0}$ & $\mathbf{1 0 0}$ \\
\hline $\mathrm{p}$-value & 0.0177 & 0.0557 & 0.0414 & 0.199 & 0.414 \\
\hline
\end{tabular}

Elaboración propia.

Excluimos las distribuciones con valores de p menores a 0.05 y aceptamos, como posibles alternativas, aquellas que tengan como mínimo un valor de 0.10. Dado lo insignificante de la diferencia del tiempo de ejecución de la simulación entre 90 y 100 horas, se elige la distribución con 100 horas. No solo porque es mejor en términos de simetría respecto a la media, sino también porque posee un mejor ajuste: $0.414>0.199$. La distribución elegida está conformada por un conjunto de valores que se ajustan a la distribución de probabilidad normal, se cumple el 
teorema del límite central y será posible realizar inferencia estadística a nuestro indicador, para un intervalo de [93.9, 100.6] autos atendidos por el auxilio mecánico con $95 \%$ de confianza.

\section{Escenario 2}

Se desea experimentar situaciones críticas del sistema, cuando presenta niveles altos de estrés y realizar la medición de indicadores de desempeño. En este sentido, se plantea simular la posibilidad de cerrar la "Salida 1" en la dirección 2, con la finalidad de medir el nivel de congestionamiento en el tráfico, en el segundo tramo de dicha vía.

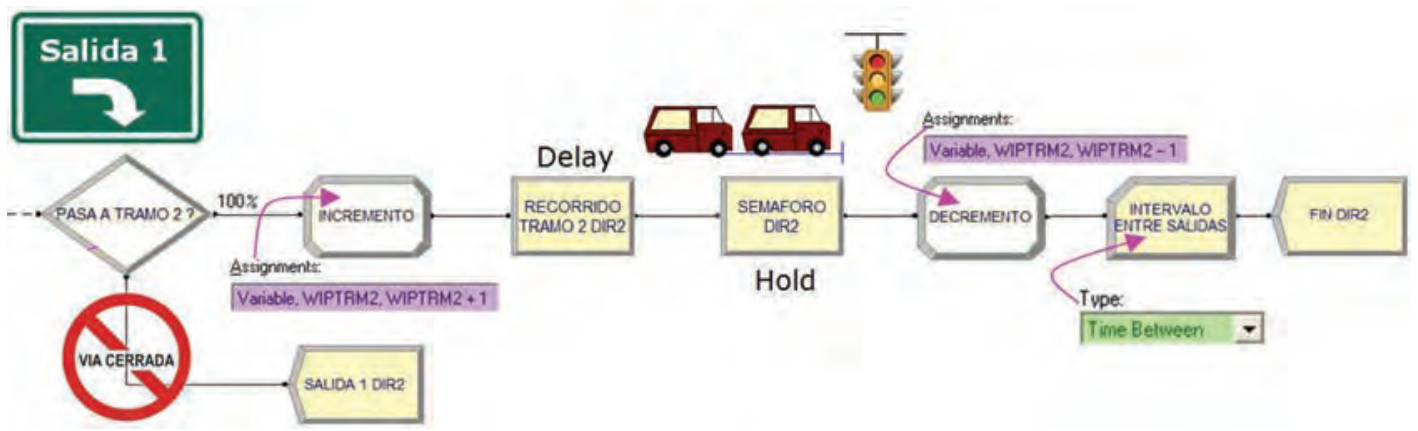

Elaboración propia con el software Arena.

Figura 32

Medición de indicadores: Escenario 2

\begin{tabular}{|c|c|c|c|c|c|}
\hline \multicolumn{6}{|c|}{ Statsic -Ádvanced Process } \\
\hline & Name & Type & Expiession & Report Label & Output File \\
\hline 1 & NUM PROM AUTOS TRAMO2 & Output & DAVG(WIPTRM2 Value) & NUM PROM AUTOS TRAMO2 & C:IWIP ESC2.DAT \\
\hline 2 & TEMPO PROM ENTRE SALDAS & Output & TAVG(INTERVALO ENTRE SALDAS) & TIEMPO PROM ENTRE SALDAS & C.ITBETWEEN ESC2.DAT \\
\hline
\end{tabular}

Fuente: Software Arena. 
Figura 33

Intervalo de confianza para los indicadores medidos

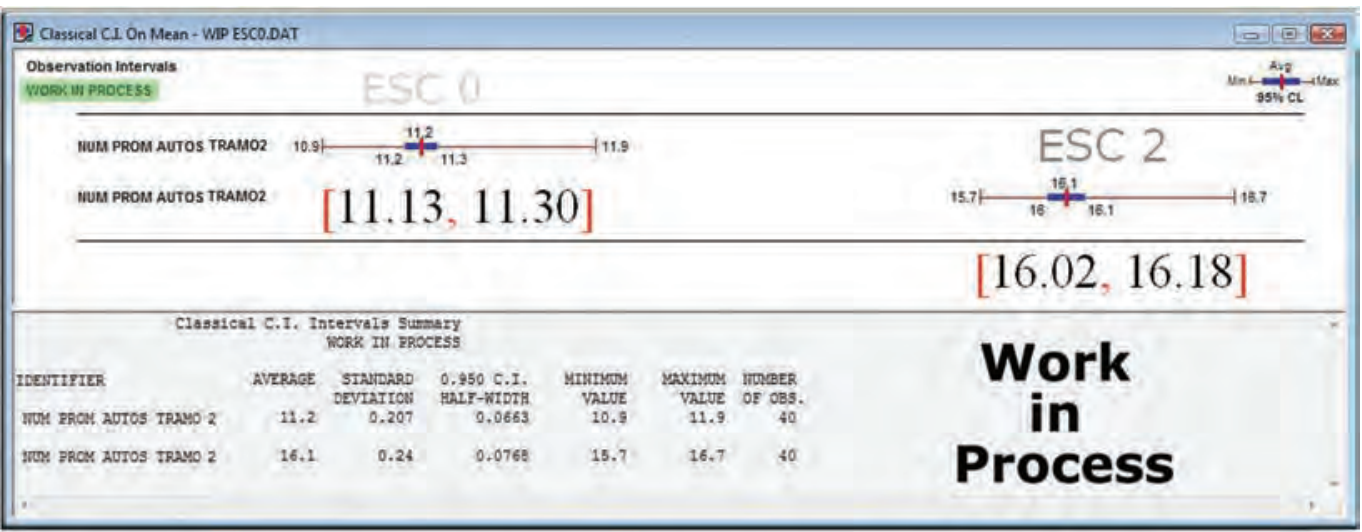

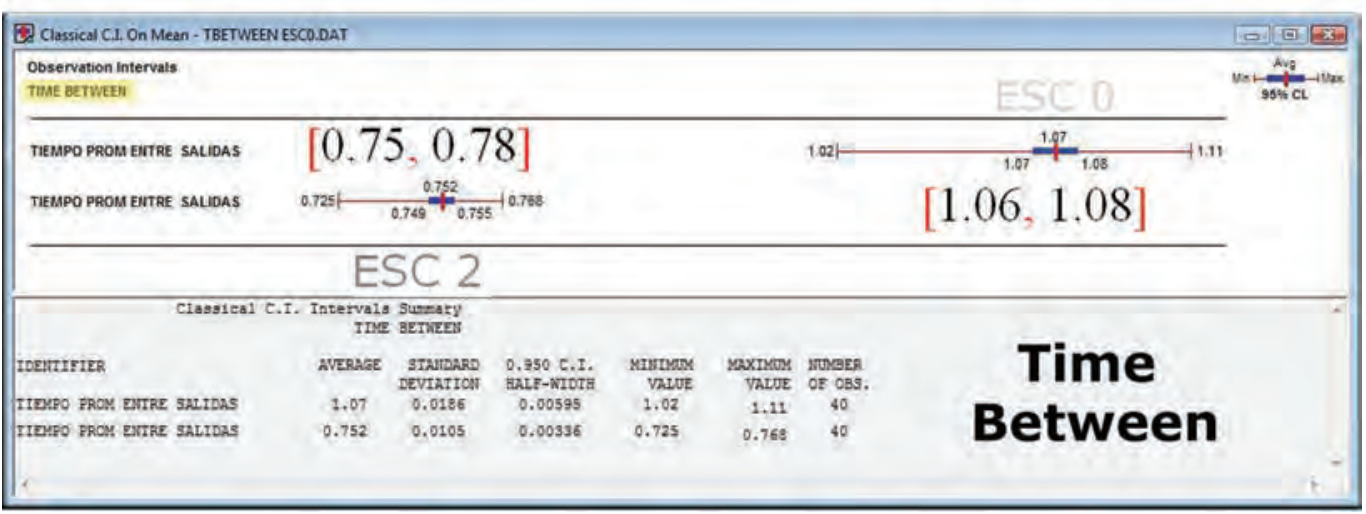

Fuente: Software Arena.

\section{Conclusiones:}

Se simuló el sistema en una posible situación crítica, cerrando la única salida lateral en la dirección 2 . Se midieron variables de interés, tales como el número promedio de autos (wip tramo 2) y el intervalo promedio entre salidas (time between). La salida lateral en la dirección 2 es relevante. Con un n.c. de $95 \%$ se obtienen los intervalos siguientes:

\begin{tabular}{|l|c|c|}
\hline & Situación normal & Situación crítica \\
\hline Work in process: & {$[11.13,11.30]$ autos } & {$[16.02,16.18]$ autos } \\
\hline Time between: & {$[1.06,1.08]$ minutos } & {$[0.75,0.78]$ minutos } \\
\hline
\end{tabular}


$\mathrm{Al}$ cerrar la salida lateral, el tráfico se incrementó de manera considerable; el número promedio de autos en el tramo 2 aumentó en $44 \%$. En cuanto al segundo indicador, se incrementó el ritmo de salida de los autos en la dirección 2; el tiempo promedio entre la salida de un auto y otro disminuyó en $30 \%$. Se tomó como referencia en el análisis el tamaño promedio de cola (number waiting semáforo 2). Si bien hubo un incremento, el valor promedio sigue siendo bajo, menor a 1: [0.31, 0.32] y $[0.44,0.45]$ autos.

\section{Escenario 3}

Dado un posible congestionamiento vehicular en la vía expresa, se desea experimentar posibles medidas con el fin de evitar que sucedan los accidentes por exceso de velocidad. Al cerrar la salida lateral en la dirección 2 se incrementó el tráfico en el tramo 2. Se desea monitorear el tráfico para controlar y reasignar la velocidad de un auto, en esta situación crítica, como se muestra en el cuadro 5.

\section{Cuadro 5}

Reasignación de velocidades

\begin{tabular}{|l|l|c|c|}
\hline \multirow{2}{*}{$\begin{array}{c}\text { Velocidad } \\
\text { de ingreso }\end{array}$} & \multicolumn{3}{|c|}{$\begin{array}{c}\text { Control de velocidad } \\
\text { Dirección 2 - Tramo 2 }\end{array}$} \\
\cline { 2 - 4 } V. Baja & Wip > 20 & Wip > 15 & Wip < 15 \\
\hline V. Media & Reducir a v. baja & Mantener velocidad & Mantener velocidad \\
\hline V. Alta & Reducir a v. baja & Reducir a v. media & Mantener velocidad \\
\hline
\end{tabular}

Elaboración propia.

De acuerdo a la situación descrita, es necesario observar el tráfico en la zona afectada y monitorear la variable work in process, es decir el número de autos en dicha zona. Dependiendo del nivel de congestionamiento del tráfico se controlará la velocidad de los autos ingresantes en base a la tabla propuesta. Los cambios realizados al modelo y las variables a medir se muestran en las figuras 34 y 35 : 
Figura 34

Cambios en el modelo cuando se reasignan velocidades: Escenario 3

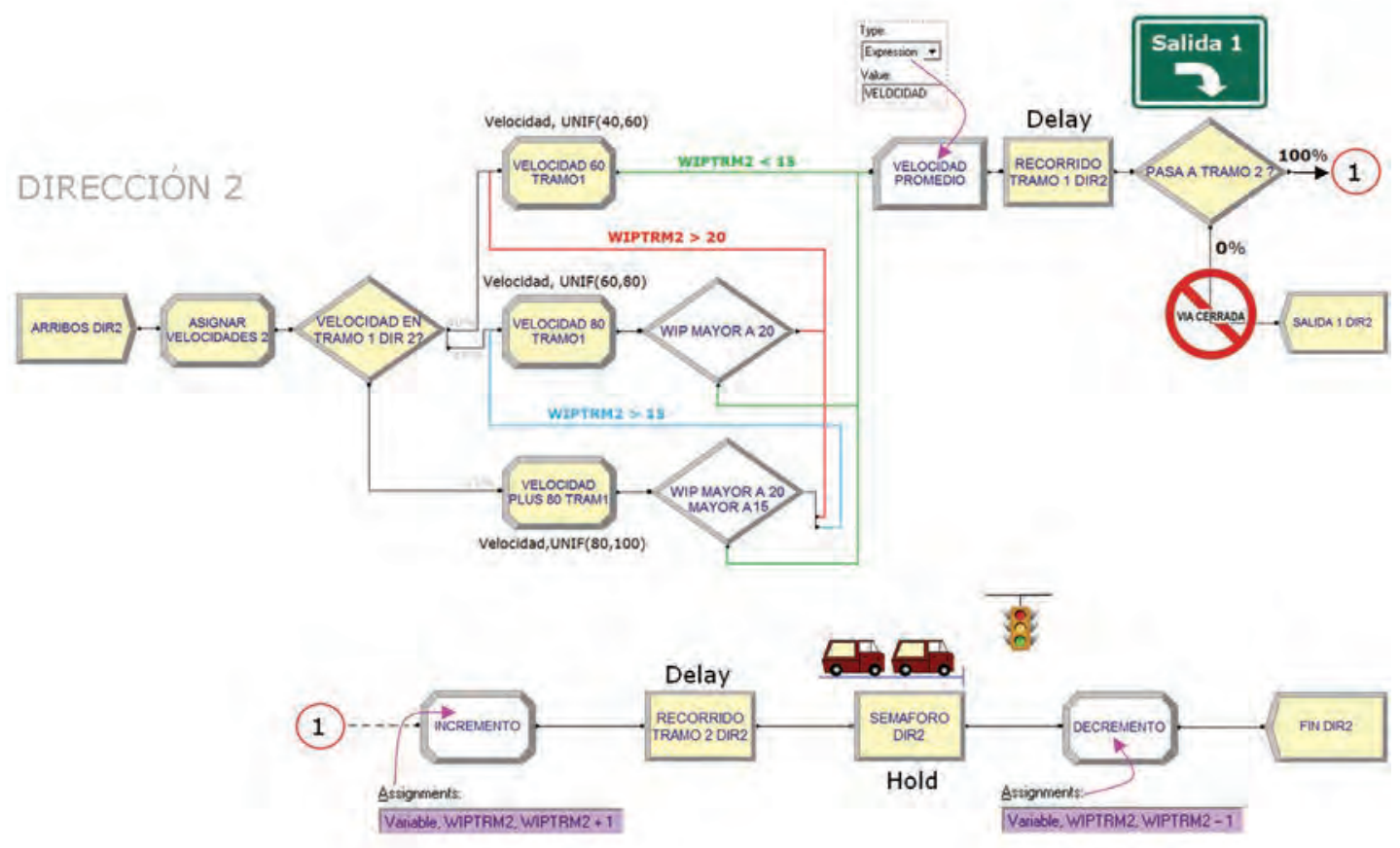

Elaboración propia con el software Arena.

Figura 35

Medición de indicadores: Escenario 3

\begin{tabular}{|c|c|c|c|c|}
\hline \multicolumn{5}{|c|}{ Statistic-Advanced Process: } \\
\hline & Name & Type & Expression & Output File \\
\hline 1 & NUM PROIM AUTOS TRAMO 2 & Output & DAVG(WIPTRM2 Value) & C:IWIP ESC3.DAT \\
\hline 2 & TIEMPO PROM ENTRE SALIDAS & Output & TAVG(INTERVALO ENTRE SALIDAS) & C.ITBETWEEN ESC3.DAT \\
\hline 3 & NUM MAX AUTOS TRAMO 2 & Output & DMAX(WIPTRM2 Value) & C:MAX ESC3.DAT \\
\hline 4 & VELOCIDAD PROMEDIO TRAMO 1 & Output & TAVG(VELOCIDAD PROMEDIO) & C:IVELOCDAD PROMEDIO.DAT \\
\hline
\end{tabular}

Elaboración propia con el software Arena. 
Figura 36

Intervalos de confianza para los indicadores medidos

\begin{tabular}{l}
$\begin{array}{l}\text { Velocidad Promedio } \\
\text { Tramo 1 }\end{array}$ \\
$\begin{array}{l}\text { Núm Prom Autos } \\
\text { Tramo 2 }\end{array}$ \\
$\begin{array}{l}\text { Núm Máx Autos } \\
\text { Tramo 2 }\end{array}$ \\
$\begin{array}{l}\text { Tiempo Promedio } \\
\text { entre Salidas }\end{array}$ \\
\hline
\end{tabular}

Fuente: Software Arena.

\section{Conclusiones:}

Se reformuló el modelo original, cerrando la salida lateral en la dirección 2 y se modeló la posibilidad de que, dependiendo del nivel de congestión en el tráfico en el tramo 2 , se pueda modificar el tiempo de viaje y controlar (o disminuir) la velocidad de aquellos autos que excedan los límites propuestos. En el cuadro 6 se presenta la matriz comparativa que se obtuvo midiendo diversos indicadores de desempeño, con un nivel de confianza de $95 \%$.

\section{Cuadro 6}

Matriz comparativa de resultados

\begin{tabular}{|l|c|c|c|}
\hline \multirow{2}{*}{ Indicador } & \multicolumn{3}{|c|}{ Dirección 2} \\
\cline { 2 - 4 } & $\begin{array}{c}\text { Situación } \\
\text { normal }\end{array}$ & $\begin{array}{c}\text { Situación } \\
\text { crítica }\end{array}$ & $\begin{array}{c}\text { Situación crítica } \\
\text { controlada }\end{array}$ \\
\hline Velocidad promedio (tramo 1) & {$[64.9,65.1]$} & {$[64.9,65.1]$} & {$[59.9,60.2]$} \\
\hline Número promedio autos (tramo 2) & {$[11.18,11.32]$} & {$[15.98,16.14]$} & {$[17.10,17.30]$} \\
\hline Número máximo autos (tramo 2) & {$[24.4,25.3]$} & {$[31.9,33.2]$} & {$[37.9,11.32]$} \\
\hline Intervalo entre salidas (tramo 2) & {$[1.06,1.08]$} & {$[0.75,0.76]$} & {$[0.75,0.76]$} \\
\hline
\end{tabular}

Elaboración propia. 
Como se observa en el cuadro 6, la velocidad promedio en el tramo 1 en una situación crítica se mantiene. Sin embargo, al monitorear el tráfico (controlando el indicador wip) se logra el objetivo de reducir la velocidad promedio de los autos que llegan a la zona de congestión, disminuyendo así la posibilidad de accidentes. En una situación controlada, los autos disminuyen la magnitud de la velocidad en un 8\% (a $60 \mathrm{~km} / \mathrm{h}$ ).

En una situación crítica controlada, al haber congestión se disminuye la velocidad y se incrementan los tiempos de viaje, entonces el wip tiende a subir. Ello explica las diferencias significativas, tanto en el número promedio de autos (wip) como en el número máximo de autos (véase el cuadro comparativo). Con un nivel de confianza de $95 \%$, se concluye que no existe diferencia estadísticamente significativa en el intervalo promedio entre salidas, entre una situación crítica controlada y una no controlada. Intervalo obtenido en la comparación de medias: $[-0.0012,+0.0086]$ minutos.

Finalmente, sería interesante analizar la posibilidad de agregar otros ingresos a la vía expresa, así como evaluar la posibilidad de incluir, modelar semáforos en las salidas laterales de la vía, medir el nivel de embotellamiento y analizar la problemática desde el punto de vista macroscópico, es decir, considerando el flujo de los vehículos en la vía expresa.

\section{REFERENCIAS}

1. Allende, H. (2002). Test de hipótesis. Recuperado de http:www. inf.utfsm.cl/ hallende/Patern/ apuntes/Cap00Simulacion.pdf

2. Banks, J. (2004). Discrete-event system simulation. En: W. J. Fabrycky \& J. H. Mize (Eds.). Prentice Hall International, series in industrial and systems ingineering, 354-382. Nueva Jersey: Series Editors.

3. Chanca, C. J. \& Castellanos, J. (2004). Simulación microscópica de tráfico urbano y su aplicación en un área de la ciudad de Zaragoza. Recuperado de http://www.mendeley.com/research/ simulacin-microscpica-trfico-urbano-y-su/\#page-1. 
4. Guasch, P. A., Piera, M., Casanovas J. \& Figueras, F. (2002). Modelado y simulación. Aplicación a procesos logísticos de fabricación y servicios. Recuperado de http://www.edicionsupc. es/ftppublic/pdfmostra/EE04603M.pdf

5. Harrell, CH., \& Tumay, K. (1995). Simulation made easy. A Manager's Guide, 115-134. Georgia: Industrial Engineers \& Management Press.

6. Harrell, Ch., Ghosh, B. K., \& Bowden, R. (2000). Simulation using ProModel. Boston: McGraw-Hill Higher Education.

7. Hoeger, H. (2011). Simulación. Web del profesor (pp. I-1/I-18). Recuperado de http://webdelprofesor.ula.ve/ingenieria/hhoeger/ simulacion/PARTE1.pdf

8. Kelton, W. D., Sadowski, R. P., \& Sturrock, D. T. (2004). Simulation with Arena. Nueva York: McGraw-Hill Higher Education.

9. Low, A. M. (1983). Statistical analysis of simulation output data. Arizona: Informs.

10. Mauttone, A., Urquhart, M., \& Alaggia, S. (2010). Simulación a eventos discretos. Recuperado de http:www.fing.edu.uy/inco/cursos/ simulación.

11. Pritsker, A. A. B. \& O’Reilly, J. J. (1999). Simulation with Visual SLAM and AweSim. Nueva York: J. Willey \& Sons.

12. Torres Vega, P. J. (2010). Simulación de sistemas con el software Arena. Lima: Universidad de Lima, Fondo Editorial.

13. Woolfson, M. M. \& Pert, G. J. (1999). An introduction to computer simulation. Nueva York: Oxford University Press Inc. 